\title{
Lions-Peetre reiteration formulas for triples and their applications
}

\author{
by \\ Irina AsEkritova (Yaroslavl'), NAtAn KRUGLJAK (Yaroslavl'), \\ LECH Maligranda (Luleå), Lyudmila Nikolova (Sofia) \\ and LARS-ERIK PERsson (Luleå)
}

Dedicated to Professor Jaak Peetre on the occasion of his 65th birthday

\begin{abstract}
We present, discuss and apply two reiteration theorems for triples of quasiBanach function lattices. Some interpolation results for block-Lorentz spaces and triples of weighted $L_{p}$-spaces are proved. By using these results and a wavelet theory approach we calculate $(\theta, q)$-spaces for triples of smooth function spaces (such as Besov spaces, Sobolev spaces, etc.). In contrast to the case of couples, for which even the scale of Besov spaces is not stable under interpolation, for triples we obtain stability in the frame of Besov spaces based on Lorentz spaces. Moreover, by using the results and ideas of this paper, we can extend the Stein-Weiss interpolation theorem known for $L_{p}(\mu)$-spaces with change of measures to Lorentz spaces with change of measures. In particular, the results obtained show that for some problems in analysis the three-space real interpolation approach is really more useful than the usual real interpolation between couples.
\end{abstract}

0. Introduction. The absence of genuine applications and the unclear situation in the theory of real interpolation of more than two spaces (cf. Section 2) have given many people a negative attitude towards all questions related to interpolation of several spaces. The main idea of this paper is to show that at least for some problems in analysis the three-space approach can be really useful. The first problem is connected with spaces of smooth functions. For this we combine reiteration and wavelet approach from which it follows that many important Banach spaces are isomorphic to a Banach lattice. For example, Besov spaces are just isomorphic to weighted $l_{p}$ spaces with certain weights and a special measure space.

2000 Mathematics Subject Classification: 46B70, 46M35, 46E35, 46E30, 42C99.

Key words and phrases: quasi-Banach spaces, Besov spaces, Lorentz spaces, blockLorentz spaces, spaces of smooth functions, wavelets, weighted $L_{p}$-spaces, Banach function lattices, quasi-Banach function lattices, real interpolation, reiteration, stability, triples, $K$-functional. 
On the other hand, from the papers of Asekritova-Krugljak [AK] and Sparr $[\mathrm{S}]$ it follows that for a triple of Banach function lattices the analogue of the famous Lions-Peetre reiteration formula for couples

$$
\left(\bar{X}_{\theta_{0}, q_{0}}, \bar{X}_{\theta_{1}, q_{1}}\right)_{\theta, q}=\bar{X}_{(1-\theta) \theta_{0}+\theta \theta_{1}, q} \quad\left(\theta_{0} \neq \theta_{1}\right)
$$

holds. These tools are used to prove the main result of the paper:

Let $X_{0}, X_{1}, X_{2}$ be a triple of smooth function spaces, where $X_{i}$ has smoothness index $\sigma_{i}$ and integration exponent $p_{i}, i=0,1,2$ (cf. Section 5). In particular, the Besov spaces $B_{p_{i}}^{\sigma_{i}}$ and Sobolev spaces $W_{p_{i}}^{\sigma_{i}}$ have smoothness $\sigma_{i}$ and integration exponent $p_{i}, i=0,1,2$.

The main result yields: If $1<p_{0}, p_{1}, p_{2}<\infty$ and

$$
\text { the points }\left(\sigma_{i}, 1 / p_{i}\right), i=0,1,2 \text {, are not collinear, }
$$

then

$$
\left(X_{0}, X_{1}, X_{2}\right)_{\left(\theta_{1}, \theta_{2}\right), q}=B_{p, q}^{\sigma, q}
$$

where

$$
(\sigma, 1 / p)=\left(1-\theta_{1}-\theta_{2}\right)\left(\sigma_{0}, 1 / p_{0}\right)+\theta_{1}\left(\sigma_{1}, 1 / p_{1}\right)+\theta_{2}\left(\sigma_{2}, 1 / p_{2}\right)
$$

and $B_{p, q}^{\sigma, q}:=B^{\sigma, q}\left(L_{p, q}\right)$ is a generalized Besov space based on the Lorentz spaces $L_{p, q}$. As a consequence we obtain similar results for triples of Besov and Sobolev spaces. The assumption $(0.2)$ is necessary and appears as a consequence of a two-dimensional analogue of the assumption $\theta_{0} \neq \theta_{1}$ in the Lions-Peetre reiteration formula for couples (cf. (0.1) and the discussion in Section 2).

It is interesting to note that triples are important here since for couples $\left(B_{p_{0}}^{\sigma_{0}}, B_{p_{1}}^{\sigma_{1}}\right)$ of Besov spaces the real interpolation spaces $\left(B_{p_{0}}^{\sigma_{0}}, B_{p_{1}}^{\sigma_{1}}\right)_{\theta, q}$ are rather complicated and, except the diagonal case, they fall outside the scale of Besov spaces (see e.g. [P], [Pe], [MP]). Moreover, if we consider the couples $\left(B_{p_{0}}^{\sigma_{0}, q_{0}}, B_{p_{1}}^{\sigma_{1}, q_{1}}\right)$ with the third (thinner) parameters $q_{i}$, then the spaces $\left(B_{p_{0}}^{\sigma_{0}, q_{0}}, B_{p_{1}}^{\sigma_{1}, q_{1}}\right)_{\theta, q}$ will indeed depend on $q_{0}$ and $q_{1}$ (see $[\mathrm{Kr}]$ ). This means that families of smooth functions are not in general stable under real interpolation of couples and as we see from our main result in (0.3) the situation is quite different when we interpolate between triples of smooth spaces.

Another application of the analogue of the Lions-Peetre reiteration theorem for triples of Banach function lattices is connected with the Stein-Weiss interpolation theorem for $L_{p}$-spaces with different measures. In 1958 Stein and Weiss $[\mathrm{SW}]$ proved a very useful interpolation theorem, which can be written (see also [BL], Th. 5.4.1 and 5.5.1) as

$$
\left(L_{p_{0}}\left(w_{0} d \mu\right), L_{p_{1}}\left(w_{1} d \mu\right)\right)_{\theta, p}=L_{p}(w d \mu),
$$

where 


$$
\begin{gathered}
0<p_{0}, p_{1}<\infty, \quad 0<\theta<1, \quad 1 / p=(1-\theta) / p_{0}+\theta / p_{1}, \\
w=w_{0}^{(1-\theta) p / p_{0}} w_{1}^{\theta p / p_{1}} .
\end{gathered}
$$

In this connection it is natural to ask for an analogue of formula (0.5) if we replace $L_{p}$-spaces by Lorentz $L_{p, q}$-spaces. We note that the nice formula

$$
\begin{gathered}
\left(L_{p_{0}, q_{0}}\left(w_{0} d \mu\right), L_{p_{1}, q_{1}}\left(w_{1} d \mu\right)\right)_{\theta, q}=L_{p, q}(w d \mu), \\
1 / q=(1-\theta) / q_{0}+\theta / q_{1},
\end{gathered}
$$

is not true in general (cf. Counterexample 6.3). In this paper we show that in fact the space

$$
L=\left(L_{p_{0}, q_{0}}\left(w_{0} d \mu\right), L_{p_{1}, q_{1}}\left(w_{1} d \mu\right)\right)_{\theta, q}
$$

is a block-Lorentz space with norm equivalent to

$$
\left(\sum_{k \in \mathbb{Z}}\left(\left\|f \chi_{\Omega_{k}}\right\|_{L_{p, q}(w d \mu)}\right)^{q}\right)^{1 / q},
$$

where

$$
\Omega_{k}=\Omega_{k}\left(w_{0}, w_{1}\right)=\left\{x \in \Omega: 2^{k} \leq w_{0}(x) / w_{1}(x)<2^{k+1}\right\}, \quad k \in \mathbb{Z},
$$

and powers and weights are related by $(0.6)$ and $0<q_{0}, q_{1} \leq \infty, 1 / q=$ $(1-\theta) / q_{0}+\theta / q_{1}$. The appearance of blocks is essential and it is easy to see that on the space of functions which are constant on $\Omega_{k}, L$ is just the usual weighted $l_{q}$-space.

In the proof of the above statement, an essential part is to first consider a Lions-Peetre reiteration type formula for triples of Banach function lattices (cf. the second reiteration theorem in Section 2) since to calculate the space $L$ directly seems to be quite difficult or even impossible because in the description we need to take rearrangements of different measures.

This paper is organized in the following way: In Section 1 we collect some preliminaries concerning real interpolation of couples and triples. In Section 2 the Lions-Peetre reiteration formula for triples of Banach function lattices is presented and discussed. Moreover, a second reiteration theorem (Theorem 2.2) is stated. The proof of this theorem is fairly technical and for the reader's convenience the details are presented in Section 7. In Section 3 we introduce a special family of weighted $L_{p}$-spaces and prove some interpolation results concerning these spaces which are useful later on and which are also of independent interest. In Section 4 we prove our main interpolation result for triples of weighted $L_{p}$-spaces (see Theorem 4.1). By using this result, reiteration and the results from Sections 2 and 3 we can state and prove the above mentioned main result for "generalized" Besov type spaces (see Theorem 5.2). Section 6 is devoted to a generalization of the Stein-Weiss theorem to the case of Lorentz spaces. The last Section 7 is reserved for an appendix with the proof of Theorem 2.3. 
Acknowledgements. The first version of this work was done by all five participants of the seminar on interpolation theory, which was held at the Department of Mathematics, Luleå University of Technology in April 1999. This research was carried out during a two-month visit of the first and second named authors at Luleå University of Technology in September and October 1999. The research of the first and fourth named authors was supported by a grant from the Swedish Natural Science Research Council

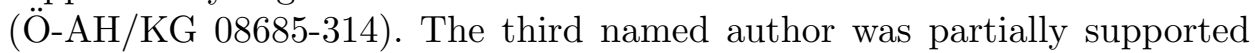
by a grant M-AA/MA 06857-306 of the Swedish Natural Science Research Council (NFR). We are grateful to the above institutions for support and to Luleå University of Technology for its hospitality.

\section{Preliminaries on real interpolation and quasi-Banach func-} tion lattices. In applications to smooth function spaces we will need to consider both the Banach and quasi-Banach spaces. We recall that a quasiBanach space $X=(X,\|\cdot\|)$ is a complete metrizable real or complex vector space whose topology is given by a quasi-norm $\|\cdot\|$ satisfying the following three conditions: $\|x\|>0, x \in X, x \neq 0 ;\|\lambda x\|=|\lambda| \cdot\|x\|, x \in X, \lambda \in \mathbb{R}$ or $\mathbb{C} ;\left\|x_{1}+x_{2}\right\| \leq C\left(\left\|x_{1}\right\|+\left\|x_{2}\right\|\right), x_{1}, x_{2} \in X$, where $C$ is a positive constant independent of $x_{1}, x_{2}$.

Let $\bar{X}=\left(X_{0}, X_{1}\right)$ be a Banach or quasi-Banach couple, i.e., there exists a Hausdorff topological vector space $\mathcal{X}$ such that $X_{i}, i=0,1$, are Banach or quasi-Banach spaces linearly and continuously embedded in $\mathcal{X}$. Then the Peetre $K$-functional is defined, for $t>0$ and $x \in X_{0}+X_{1}$, by

$$
K(t, x ; \bar{X})=\inf \left\{\left\|x_{0}\right\|_{X_{0}}+t\left\|x_{1}\right\|_{X_{1}}: x=x_{0}+x_{1}, x_{0} \in X_{0}, x_{1} \in X_{1}\right\} .
$$

The real interpolation spaces $\bar{X}_{\theta, q}$ are defined, for $0<q \leq \infty$ and $0<\theta<1$, as the set of all $x \in X_{0}+X_{1}$ for which the norms (quasi-norms)

$$
\|x\|_{\theta, q}=\left(\int_{0}^{\infty}\left(t^{-\theta} K(t, x ; \bar{X})\right)^{q} \frac{d t}{t}\right)^{1 / q}
$$

are finite, and with the usual supremum interpretation for $q=\infty$.

Let now $\bar{X}=\left(X_{0}, X_{1}, X_{2}\right)$ be a Banach or quasi-Banach triple, i.e., $X_{i}, i=0,1,2$, are Banach or quasi-Banach spaces linearly and continuously embedded in a Hausdorff topological vector space $\mathcal{X}$. Then we can analogously define the corresponding $K$-functional, for $t_{1}, t_{2}>0$ and $x \in$ $X_{0}+X_{1}+X_{2}$, by

$$
\begin{aligned}
& K\left(t_{1}, t_{2}, x ; \bar{X}\right)=\inf \left\{\left\|x_{0}\right\|_{X_{0}}+t_{1}\left\|x_{1}\right\|_{X_{1}}+t_{2}\left\|x_{2}\right\|_{X_{2}}:\right. \\
& \left.\quad x=x_{0}+x_{1}+x_{2}, x_{0} \in X_{0}, x_{1} \in X_{1}, x_{2} \in X_{2}\right\} .
\end{aligned}
$$

Let

$$
H=\left\{\left(\theta_{1}, \theta_{2}\right): \theta_{1}>0, \theta_{2}>0 \text { and } \theta_{1}+\theta_{2}<1\right\}
$$


The spaces $\bar{X}_{\bar{\theta}, q}=\bar{X}_{\left(\theta_{1}, \theta_{2}\right), q}$ (sometimes we also denote them by $\bar{X}_{\bar{\theta}, q ; K}$ or $\left.\bar{X}_{\left(\theta_{1}, \theta_{2}\right), q ; K}\right)$ are defined, for $0<q \leq \infty$ and $\bar{\theta}=\left(\theta_{1}, \theta_{2}\right) \in H$, analogously as the set of all $x \in X_{0}+X_{1}+X_{2}$ for which the quasi-norms

$$
\|x\|_{\bar{\theta}, q}=\left(\int_{0}^{\infty} \int_{0}^{\infty}\left(t_{1}^{-\theta_{1}} t_{2}^{-\theta_{2}} K\left(t_{1}, t_{2}, x ; \bar{X}\right)\right)^{q} \frac{d t_{1}}{t_{1}} \frac{d t_{2}}{t_{2}}\right)^{1 / q}
$$

are finite, and with the usual modification for $q=\infty$.

On the other hand, we let $\bar{X}_{\bar{\theta}, q ; J}=\bar{X}_{\left(\theta_{1}, \theta_{2}\right), q ; J}$ be the space of all those elements $x \in X_{0}+X_{1}+X_{2}$ which can be represented in the form

$$
\left.x=\int_{0}^{\infty} \int_{0}^{\infty} u\left(t_{1}, t_{2}\right) \frac{d t_{1}}{t_{1}} \frac{d t_{2}}{t_{2}} \quad \text { (convergence in } X_{0}+X_{1}+X_{2}\right),
$$

where $u\left(t_{1}, t_{2}\right)$ is a strongly measurable $X_{0} \cap X_{1} \cap X_{2}$-valued function and satisfies

$$
\left(\int_{0}^{\infty} \int_{0}^{\infty}\left(t_{1}^{-\theta_{1}} t_{2}^{-\theta_{2}} J\left(t_{1}, t_{2}, u\left(t_{1}, t_{2}\right) ; \bar{X}\right)\right)^{q} \frac{d t_{1}}{t_{1}} \frac{d t_{2}}{t_{2}}\right)^{1 / q}<\infty
$$

with $J\left(t_{1}, t_{2}, u ; \bar{X}\right)=\max \left(\|u\|_{X_{0}}, t_{1}\|u\|_{X_{1}}, t_{2}\|u\|_{X_{2}}\right), u \in X_{0} \cap X_{1} \cap X_{2}$.

The norm $\|x\|_{\bar{\theta}, q ; J}$ in $\bar{X}_{\bar{\theta}, q ; J}$ is the infimum of the values of the integral (1.3) over all such representations (1.2) of $x$.

Concerning $K$ - and $J$-interpolation methods between two Banach or quasi-Banach spaces we refer to the books $[\mathrm{BL}],[\mathrm{BK}]$ and [KPS], and for $K$ - and $J$-interpolation methods between three or more spaces to [S], [AK] and $[\mathrm{E}]$.

We only mention here that in contrast to the classical case of Banach or quasi-Banach couples, where $K$ - and $J$-methods coincide to within equivalence of quasi-norms, it is not true in general that $\bar{X}_{\bar{\theta}, q ; K}$ coincides with $\bar{X}_{\bar{\theta}, q ; J}$ for more than two spaces.

Let $(\Omega, \Sigma, \mu)$ be a $\sigma$-finite measure space. A Banach or quasi-Banach function lattice on $(\Omega, \mu)$ [also known as a Banach or quasi-Banach ideal space on $(\Omega, \mu)$, sometimes also called a Banach or quasi-Banach function space on $(\Omega, \mu)]$ will mean a Banach or quasi-Banach subspace $X=(X,\|\cdot\|)$ of $L^{0}$ (the space of classes of real or complex measurable functions defined on the measure space $(\Omega, \Sigma, \mu))$ which is an ideal, i.e., if $y \in X, x \in L^{0}$ and $|x| \leq|y| \mu$-a.e. on $\Omega$, then $x \in X$ and $\|x\| \leq\|y\|$.

A couple (or triple) of Banach or quasi-Banach function lattices on $\Omega$ will mean two (or three) Banach or quasi-Banach function lattices on $(\Omega, \mu)$, respectively.

Let $w$ be a fixed weight function on $\Omega$, i.e., a positive measurable function on $\Omega$. For $\sigma \in \mathbb{R}$ and $0<p \leq \infty$ we consider the family of quasi-Banach 
function lattices $L_{p}^{\sigma}=L_{p}\left(w^{\sigma}\right)$ defined by the finiteness of the quasi-norms

$$
\|f\|_{L_{p}^{\sigma}}=\left(\int_{\Omega}\left|f(x) w(x)^{\sigma}\right|^{p} d \mu\right)^{1 / p}
$$

with the usual (supremum) interpretation for $p=\infty$. The importance of this particular family of Banach (quasi-Banach in the case $p<1$ ) function lattices on $\Omega$ is that the Besov spaces $B_{p}^{\sigma}$ can be represented in terms of wavelets as a special case of such spaces (see our Section 5 ).

The weighted Lorentz spaces $L_{p, q}(w, \mu)$ on $\Omega$ (for $0<p, q \leq \infty$ and with the convention that $q=\infty$ when $p=\infty$ ) are determined by the quasi-norm

$$
\|f\|_{L_{p, q}(w, \mu)}=\left(\int_{0}^{\infty}\left(t^{1 / p}(f \cdot w)_{\mu}^{*}(t)\right)^{q} \frac{d t}{t}\right)^{1 / q}
$$

with the usual supremum modification for $q=\infty$. Here by $g_{\mu}^{*}$ we denote the decreasing rearrangement of the function $g$ on $\Omega$ with respect to the measure $\mu$.

2. On the Lions-Peetre reiteration formula for triples. In 1964 Lions and Peetre $[\mathrm{LP}]$ proved one of the most important theoretical result in interpolation theory, the so-called reiteration formula for couples of Banach spaces:

$$
\left(\bar{X}_{\theta_{0}, q_{0}}, \bar{X}_{\theta_{1}, q_{1}}\right)_{\lambda, q}=\bar{X}_{\theta, q}, \quad \theta=(1-\lambda) \theta_{0}+\lambda \theta_{1},
$$

where

$$
\theta_{0} \neq \theta_{1}
$$

This formula also holds for quasi-Banach spaces (cf. [BL], Th. 3.11.5). It shows not only the stability of the spaces $\bar{X}_{\theta, q}$ but it also gives a possibility to calculate the interpolation spaces for rather complicated couples $\left(\bar{X}_{\theta_{0}, q_{0}}, \bar{X}_{\theta_{1}, q_{1}}\right)$ by using a simpler initial couple $\bar{X}=\left(X_{0}, X_{1}\right)$ and the result does not depend on the parameters $q_{0}$ and $q_{1}$.

The classical proof of the formula (2.1) is based on the so-called equivalence theorem for the $K$ - and $J$-methods:

$$
\bar{X}_{\theta, q ; K}=\bar{X}_{\theta, q ; J}
$$

which is valid for any couple $\bar{X}=\left(X_{0}, X_{1}\right)$ of quasi-Banach spaces (cf. [BL], Th. 3.11.3). Sparr (1974) defined the $K$-, $J$-functionals and the corresponding interpolation spaces for $(n+1)$-tuples $\bar{X}=\left(X_{0}, X_{1}, \ldots, X_{n}\right), n \geq 1$, of Banach spaces which were analogous to the case of couples and showed that we always have the imbedding

$$
\bar{X}_{\bar{\theta}, q ; J} \subset \bar{X}_{\bar{\theta}, q ; K}
$$

Moreover, Sparr $[\mathrm{S}]$ tried to extend the reiteration formula $(2.1)$ to $(n+1)$ tuples and he showed that if an analogue of the equivalence theorem is 
valid for a triple $\bar{X}=\left(X_{0}, X_{1}, X_{2}\right)$, then an analogue of the Lions-Peetre reiteration formula is also true. But there are troubles with the equivalence theorem for $n>1$. We should mention that earlier Yoshikawa (1970) considered the spaces of means for $n$-tuples. Yoshikawa (1970) and Sparr (1974) gave counterexamples to the equivalence theorem which means in fact that the above imbedding is strict for $n>1$. Their counterexample could be considered somewhat "artificial" since it has the property that the intersection of the spaces is equal to $\{0\}$. In 1987 Cwikel and Janson [CJ] constructed a "non-degenerate" 3 -tuple $\bar{H}=\left(H_{0}, H_{1}, H_{2}\right)$ of Hilbert spaces with the intersection $H_{0} \cap H_{1} \cap H_{2}$ dense in each $H_{i}$ and such that the imbedding $\bar{H}_{\bar{\theta}, 2 ; J} \subset \bar{H}_{\bar{\theta}, 2 ; K}$ is strict, i.e., the equivalence theorem is not valid. Thus we conclude that even for a good triple, such as a triple of Hilbert spaces, the classical method of proving the reiteration theorem does not work.

There was another way to prove the Lions-Peetre reiteration formula based on the $K$-divisibility property of couples (cf. [BK], Th. 3.3.11). The well known Brudnyı̄-Krugljak result from 1981 states that any Banach or quasi-Banach couple is $K$-divisible but as observed by Asekritova [A] in 1988 the $K$-divisibility property is not valid for triples, even for simple triples such as $\left(L_{1}, L_{1}\left(1 / t_{1}\right), L_{1}\left(1 / t_{2}\right)\right)$.

These examples gave an impression that an analogue of the reiteration theorem for triples was valid only for a very narrow class of triples, and

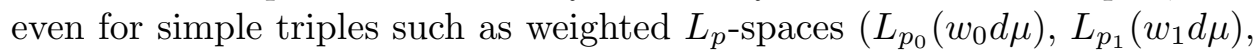
$\left.L_{p_{2}}\left(w_{2} d \mu\right)\right)$ it was not clear if it was true or not. This unclear situation and absence of genuine applications has made people rather pessimistic about real interpolation of several spaces.

In this situation it was natural to try to prove that the reiteration theorem can be valid for wide and important classes of triples and to find interesting applications.

In 1997 Asekritova and Krugljak [AK] showed that the equivalence theorem is in fact valid for any $n$-tuple of Banach function lattices on $\Omega$. In particular, it holds for triples $\left(L_{p_{0}}\left(w_{0} d \mu\right), L_{p_{1}}\left(w_{1} d \mu\right), L_{p_{2}}\left(w_{2} d \mu\right)\right)$ of weighted $L_{p}$-spaces.

The proof of Asekritova-Krugljak's result is rather complicated and it uses significantly the structure of Banach function lattices on $\Omega$. Their theorem for triples $\left(X_{0}, X_{1}, X_{2}\right)$ of Banach function lattices on $\Omega$, together with Sparr's theorem ([S], Th. 9.1, p. 285) for $n=2$ give the reiteration theorem in the case of Banach function lattices. Moreover, from these investigations it can be seen that it is enough to require that $X_{i}, i=0,1,2$, are quasi-Banach function lattices on $\Omega$ so from $[\mathrm{AK}]$ and $[\mathrm{S}]$ we can derive the following first reiteration theorem (this theorem is valid even for $n$-tuples but for our purpose it is enough to have the simplest case of triples): 
Theorem 2.1 (First Reiteration Theorem). Let $\bar{X}=\left(X_{0}, X_{1}, X_{2}\right)$ be a triple of Banach or quasi-Banach function lattices on $\Omega$ and let $\bar{\lambda}=$ $\left(\lambda_{1}, \lambda_{2}\right) \in H, \bar{\theta}_{i}=\left(\theta_{1}^{i}, \theta_{2}^{i}\right) \in H, i=0,1,2$. Then

$$
\left(\bar{X}_{\bar{\theta}_{0}, q_{0}}, \bar{X}_{\bar{\theta}_{1}, q_{1}}, \bar{X}_{\bar{\theta}_{2}, q_{2}}\right)_{\bar{\lambda}, q}=\bar{X}_{\bar{\theta}, q}, \quad \bar{\theta}=\left(1-\lambda_{1}-\lambda_{2}\right) \bar{\theta}_{0}+\lambda_{1} \bar{\theta}_{1}+\lambda_{2} \bar{\theta}_{2}
$$
whenever the vectors $\bar{\theta}_{0}, \bar{\theta}_{1}, \bar{\theta}_{2}$ are not collinear.

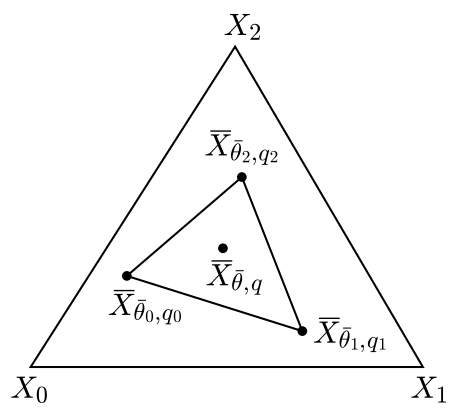

Fig. 1

The formula (2.4) has a simple geometrical interpretation. Let us draw a triangle $\Delta$ in the plane (see the outer triangle in Figure 1 ) corresponding to the triple $\bar{X}=\left(X_{0}, X_{1}, X_{2}\right)$. Identify the space $\bar{X}_{\left(\theta_{1}, \theta_{2}\right), q}$ with the point of $\Delta$ with barycentric coordinates $\left(1-\theta_{1}-\theta_{2}, \theta_{1}, \theta_{2}\right)$. Then the triple $\left(\bar{X}_{\bar{\theta}_{0}, q_{0}}, \bar{X}_{\bar{\theta}_{1}, q_{1}}, \bar{X}_{\bar{\theta}_{2}, q_{2}}\right)$ corresponds to a triangle $\Delta^{\prime}$ inside $\Delta$. If $B$ is a fixed point in $\Delta^{\prime}$, then the following two spaces correspond to it:

$$
\left(X_{0}, X_{1}, X_{2}\right)_{\left(\theta_{1}, \theta_{2}\right), q} \text { from the initial triple }\left(X_{0}, X_{1}, X_{2}\right)
$$

and

$$
\begin{aligned}
\left(\bar{X}_{\bar{\theta}_{0}, q_{0}}, \bar{X}_{\bar{\theta}_{1}, q_{1}}, \bar{X}_{\bar{\theta}_{2}, q_{2}}\right)_{\left(\lambda_{1}, \lambda_{2}\right), q} & \text { from the other triple }\left(\bar{X}_{\bar{\theta}_{0}, q_{0}}, \bar{X}_{\bar{\theta}_{1}, q_{1}}, \bar{X}_{\bar{\theta}_{2}, q_{2}}\right) .
\end{aligned}
$$

The First Reiteration Theorem states that these two spaces coincide and the parameters are connected by the usual geometrical relations for barycentric coordinates.

REMARK 2.2. (a) The condition (2.5) is just the analogue of the necessary condition $\theta_{0} \neq \theta_{1}$ for the reiteration formula (2.1) for couples to hold and it means that the vertices of the triangle $\Delta^{\prime}$ are not collinear.

(b) In the definition of the spaces $\bar{X}_{\left(\theta_{1}, \theta_{2}\right), q}$ we supposed that $\theta_{0}, \theta_{1}>0$ and $\theta_{0}+\theta_{1}<1$. It is possible to give a meaning to these spaces also for $\theta_{0}, \theta_{1} \geq 0, \theta_{0}+\theta_{1} \leq 1$ and then the reiteration formula (2.4) will be valid even in such cases (see $[\mathrm{S}]$ and $[\mathrm{AK}]$ ). 
We will also need the following theorem which shows that in some cases the space obtained by successive interpolation of couples can be obtained by interpolation of triples. We will call this theorem the second reiteration theorem.

Theorem 2.3 (Second Reiteration Theorem). Let $\left(X_{0}, X_{1}, X_{2}\right)$ be a triple of Banach or quasi-Banach function lattices on $\Omega$. If $0<q_{0}, q_{1}, q<\infty$ and $1 / q=(1-\mu) / q_{0}+\mu / q_{1}$, then

$$
\left(\left(X_{0}, X_{2}\right)_{\alpha_{0}, q_{0}},\left(X_{1}, X_{2}\right)_{\alpha_{1}, q_{1}}\right)_{\mu, q}=\left(X_{0}, X_{1}, X_{2}\right)_{\left(\theta_{1}, \theta_{2}\right), q},
$$
where

$$
\theta_{1}=\left(1-\alpha_{1}\right) \mu, \quad \theta_{2}=\alpha_{0}(1-\mu)+\alpha_{1} \mu
$$

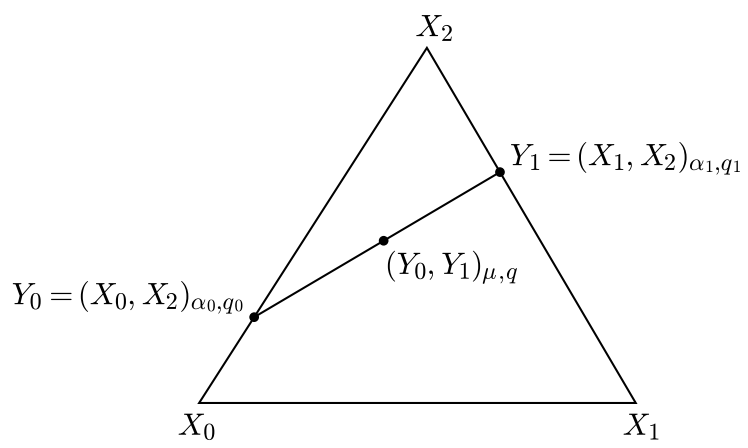

Fig. 2

The proof of Theorem 2.3 is rather long and it will be given, for the convenience of the reader, in Appendix 7.

REMARK 2.4. For the case of $q_{0}=q_{1}, \alpha_{0}=\alpha_{1}$ and for a triple of Banach spaces for which the equivalence theorem holds, the result of Theorem 2.3 was also pointed out by Yoshikawa [Y], Prop. 4.1, 4.2 (see also Sparr [S], Th. 9.3, p. 292, and in the particular case $n=2, m=1$, p. 291).

REMARK 2.5. In the case when $\alpha_{1}=0$, i.e., when instead of the space $\left(X_{1}, X_{2}\right)_{\alpha_{1}, q_{1}}$ we have the space $X_{1}$, then the formula (2.6) is not true in general. In fact, in Example 4.6 we will consider the triple of weighted $L_{p^{-}}$ spaces for weighted spaces $X_{0}, X_{1}, X_{2}$ such that

$$
\left(\left(X_{0}, X_{2}\right)_{\alpha_{0}, q_{0}}, X_{1}\right)_{\mu, q} \neq\left(X_{0}, X_{1}, X_{2}\right)_{\left(\mu, \alpha_{0}(1-\mu)\right), q} .
$$

Note that in Theorem 2.3 the second space in the couples $\left(X_{0}, X_{2}\right)$ and $\left(X_{1}, X_{2}\right)$ is the same space, and this is essential since the formula

$$
\left(\left(X_{0}, X_{2}\right)_{\alpha_{0}, q_{0}},\left(X_{1}, X_{3}\right)_{\alpha_{1}, q_{1}}\right)_{\mu, q}=\left(X_{0}, X_{1}, X_{2}, X_{3}\right)_{\left(\left(1-\alpha_{1}\right) \mu, \alpha_{0}(1-\mu), \alpha_{1} \mu\right), q}
$$

is not true in general (the definition of real interpolation spaces between four spaces is similar to that for three spaces, see e.g. Sparr $[\mathrm{S}]$ ). Indeed, if 
we take $X_{1}=X_{3}$, then

$$
\left(X_{0}, X_{1}, X_{2}, X_{3}\right)_{\left(\left(1-\alpha_{1}\right) \mu, \alpha_{0}(1-\mu), \alpha_{1} \mu\right), q}=\left(X_{0}, X_{1}, X_{2}\right)_{\left(\mu, \alpha_{0}(1-\mu)\right), q}
$$

and, as noted before, this is different from

$$
\left(\left(X_{0}, X_{2}\right)_{\alpha_{0}, q_{0}}, X_{1}\right)_{\mu, q}=\left(\left(X_{0}, X_{2}\right)_{\alpha_{0}, q_{0}},\left(X_{1}, X_{3}\right)_{\alpha_{1}, q_{1}}\right)_{\mu, q}
$$

Theorem 2.3 also gives the possibility to calculate $\left(X_{0}, X_{1}, X_{2}\right)_{\left(\theta_{1}, \theta_{2}\right), q}$ if we can calculate the interpolation spaces for some couples.

Corollary 2.6. Let $\left(X_{0}, X_{1}, X_{2}\right)$ be a triple of Banach or quasiBanach function lattices on $\Omega$ and $0<q<\infty$. Then

$$
\left(X_{0}, X_{1}, X_{2}\right)_{\left(\theta_{1}, \theta_{2}\right), q}=\left(\left(X_{0}, X_{2}\right)_{\theta_{2}, q},\left(X_{1}, X_{2}\right)_{\theta_{2}, q}\right)_{\theta, q},
$$

where $\theta=\theta_{1} /\left(1-\theta_{2}\right)$.

This follows immediately from Theorem 2.3 by putting $\alpha_{0}=\alpha_{1}=\theta_{2}$, $q_{1}=q_{2}=q$ and $\mu=\theta_{1} /\left(1-\theta_{2}\right)$.

We finish this section with an example which shows that even for rather simple and important triples the reiteration theorems above are not valid in general.

EXAMPLE 2.7. Let us call a triple $\left(X_{0}, X_{1}, X_{2}\right)$ "good" if both our reiteration theorems (the first and second) are true for it. Then the triple $\left(\stackrel{\circ}{W}_{2}^{1}, W_{2}^{1}, L_{2}\right)$ is not good, where all spaces consist of functions on the disc $D=\left\{(x, y): x^{2}+y^{2}<1\right\}$ and $\stackrel{\circ}{W}_{2}^{1}$ is the closure in $W_{2}^{1}$ of the $C^{\infty}$ functions with a compact support.

Indeed, the proof of statement (a) of Theorem 3 in $[\mathrm{AK}]$ shows that if the triple $\left(X_{0}, X_{1}, X_{2}\right)$ is good and the equality

$$
\left(X_{0}, X_{2}\right)_{\theta, q}=\left(X_{1}, X_{2}\right)_{\theta, q}
$$

holds for some parameters $\theta, q$, then it holds for all $\theta, q$. However, according to a result of Lions-Magenes (see $[\mathrm{LM}]$, Th. 11.1, p. 55) we have

$$
\left(\stackrel{\circ}{W}_{2}^{1}, L_{2}\right)_{\theta, 2}=\left(W_{2}^{1}, L_{2}\right)_{\theta, 2} \quad \text { for } 1 / 2<\theta<1
$$

but

$$
\left(\stackrel{\circ}{W}_{2}^{1}, L_{2}\right)_{\theta, 2} \neq\left(W_{2}^{1}, L_{2}\right)_{\theta, 2} \quad \text { for } 0<\theta \leq 1 / 2
$$

which shows that the triple $\left(\stackrel{\circ}{W}_{2}^{1}, W_{2}^{1}, L_{2}\right)$ is not good.

This example once again shows the importance of a common order structure of the spaces. This situation was also observed, for example, by Maligranda $[\mathrm{M}]$ who showed that the equality

$$
\left(X_{0}, X_{1} \cap X_{2}\right)_{\theta, q}=\left(X_{0}, X_{1}\right)_{\theta, q} \cap\left(X_{0}, X_{2}\right)_{\theta, q}
$$

is true for Banach function lattices but, as was known from counterexamples, not for general Banach spaces. 
3. Interpolation of a family of block-Lorentz spaces. Let $w$ be a fixed weight function on $\Omega$ and consider the weighted spaces $L_{p}^{\sigma}=L_{p}\left(w^{\sigma}\right)$, for $\sigma \in \mathbb{R}$ and $0<p \leq \infty$, on $\Omega$ defined by the finiteness of the quasi-norms (1.4). Then, according to the Stein-Weiss interpolation theorem (see [BL], Th. 5.5.1), in the diagonal case, i.e., when $1 / p=(1-\theta) / p_{0}+\theta / p_{1}$, we have

$$
\left(L_{p_{0}}^{\sigma_{0}}, L_{p_{1}}^{\sigma_{1}}\right)_{\theta, p}=L_{p}^{\sigma}, \quad \sigma=(1-\theta) \sigma_{0}+\theta \sigma_{1} .
$$

If we now identify the spaces $L_{p_{i}}^{\sigma_{i}}$ with the points $\left(\sigma_{i}, 1 / p_{i}\right), i=0,1$, in the upper half-plane, then the interpolation space $L_{p}^{\sigma}$ will be identified with the point $(\sigma, 1 / p)$ on the straight line between $\left(\sigma_{0}, 1 / p_{0}\right)$ and $\left(\sigma_{1}, 1 / p_{1}\right)$.

Let us now introduce the sets

$$
\Omega_{k}=\Omega_{k}(w)=\left\{x \in \Omega: 2^{k} \leq w(x)<2^{k+1}\right\}, \quad k \in \mathbb{Z},
$$

and define, for $\sigma \in \mathbb{R}$ and $0<p, q, r \leq \infty$, the block-Lorentz spaces $L_{p, r}^{\sigma, q}=$ $L_{p, r}^{\sigma, q}(w)$ by the finiteness of the quasi-norm

$$
\|f\|_{L_{p, r}^{\sigma, q}}=\left(\sum_{k \in \mathbb{Z}}\left(\left\|f w^{\sigma} \chi_{\Omega_{k}}\right\|_{L_{p, r}}\right)^{q}\right)^{1 / q}
$$

with the standard modification for $q=\infty$. Here, $L_{p, r}$ denotes the usual Lorentz spaces (with the convention that $r=\infty$ when $p=\infty$ ). It is clear that $L_{p, p}^{\sigma, p}=L_{p}^{\sigma}$ and in what follows we also use the notation $L_{p}^{\sigma, q}:=L_{p, p}^{\sigma, q}$.

In the case when $r=p$ and $w(x)=|x|$ on $\mathbb{R}^{n} \backslash\{0\}$, the spaces $L_{p, r}^{\sigma, q}$ are the so-called homogeneous Herz spaces $K_{p}^{\sigma, q}$ (cf. Herz $[\mathrm{H}]$ and BaernsteinSawyer $[\mathrm{BS}]$ ).

According to the next lemma (due to Gilbert [G], Th. 3.7), for $p_{0}=$ $p_{1}=p$ we can calculate the interpolation spaces $\left(L_{p_{0}}^{\sigma_{0}}, L_{p_{1}}^{\sigma_{1}}\right)_{\theta, q}$ even in the non-diagonal case.

LEMma 3.1. If $\sigma_{0} \neq \sigma_{1}$, then

$$
\left(L_{p}^{\sigma_{0}}, L_{p}^{\sigma_{1}}\right)_{\theta, q}=L_{p}^{\sigma, q}, \quad \text { where } \sigma=(1-\theta) \sigma_{0}+\theta \sigma_{1} .
$$

Proof. For the reader's convenience we give another and, in our opinion, clearer proof than that in [G]. It is enough to consider the case $\sigma_{1}>\sigma_{0}$. The other case is obtained by just interchanging the spaces.

Set

$$
a_{k}=\left(\int_{\Omega_{k}}\left|f(x) w(x)^{\sigma_{0}}\right|^{p} d \mu\right)^{1 / p}, \quad k \in \mathbb{Z} .
$$

According to the definition of $\Omega_{k}$ we have the equivalence

$$
w(x)^{\sigma_{1}-\sigma_{0}} \approx 2^{k\left(\sigma_{1}-\sigma_{0}\right)} \quad \text { for } x \in \Omega_{k},
$$

or more precisely the estimates

$$
2^{k\left(\sigma_{1}-\sigma_{0}\right)} \leq w(x)^{\sigma_{1}-\sigma_{0}} \leq 2^{(k+1)\left(\sigma_{1}-\sigma_{0}\right)} \quad \text { for } x \in \Omega_{k} .
$$


By using these estimates we find that

$$
\begin{aligned}
\|f\|_{L_{p}^{\sigma_{1}}} & =\left(\sum_{k \in \mathbb{Z}} \int_{\Omega_{k}}\left|f(x) w(x)^{\sigma_{0}}\right|^{p} w(x)^{\left(\sigma_{1}-\sigma_{0}\right) p} d \mu\right)^{1 / p} \\
& \geq\left(\sum_{k \in \mathbb{Z}}\left(2^{k\left(\sigma_{1}-\sigma_{0}\right)} a_{k}\right)^{p}\right)^{1 / p}=\left\|\left\{a_{k}\right\}\right\|_{l_{p}^{\sigma_{1}-\sigma_{0}}}
\end{aligned}
$$

and

$$
\|f\|_{L_{p}^{\sigma_{1}}} \leq 2^{\sigma_{1}-\sigma_{0}}\left\|\left\{a_{k}\right\}\right\|_{l_{p}^{\sigma_{1}-\sigma_{0}}}
$$

where

$$
\left\|\left\{a_{k}\right\}\right\|_{l_{p}^{s}}:=\left(\sum_{k \in \mathbb{Z}}\left(2^{k s}\left|a_{k}\right|\right)^{p}\right)^{1 / p} \quad \text { for } s \in \mathbb{R} \text { and } 0<p \leq \infty .
$$

The above estimates give

$$
K\left(t, f ; L_{p}^{\sigma_{0}}, L_{p}^{\sigma_{1}}\right) \approx K\left(t,\left\{a_{k}\right\} ; l_{p}, l_{p}^{\sigma_{1}-\sigma_{0}}\right) .
$$

In fact, if $f=f_{0}+f_{1}$ with $f_{i} \in L_{p}^{\sigma_{i}}(i=0,1)$, then for

$$
a_{k, i}=\left(\int_{\Omega_{k}}\left|f_{i}(x) w(x)^{\sigma_{0}}\right|^{p} d \mu\right)^{1 / p},
$$

we have $\left\{a_{k, 0}\right\} \in l_{p},\left\{a_{k, 1}\right\} \in l_{p}^{\sigma_{1}-\sigma_{0}}$ and $a_{k} \leq 2^{\max (0,1 / p-1)}\left(a_{k, 0}+a_{k, 1}\right)$ for all $k \in \mathbb{Z}$. Hence

$$
\begin{aligned}
K\left(t,\left\{a_{k}\right\} ; l_{p}, l_{p}^{\sigma_{1}-\sigma_{0}}\right) & \leq 2^{\max (0,1 / p-1)}\left(\left\|\left\{a_{k, 0}\right\}\right\|_{l_{p}}+t\left\|\left\{a_{k, 1}\right\}\right\|_{l_{p}^{\sigma_{1}-\sigma_{0}}}\right) \\
& \leq 2^{\max (0,1 / p-1)}\left(\left\|f_{0}\right\|_{L_{p}^{\sigma_{0}}}+t\left\|f_{1}\right\|_{L_{p}^{\sigma_{1}}}\right)
\end{aligned}
$$

and so

$$
K\left(t,\left\{a_{k}\right\} ; l_{p}, l_{p}^{\sigma_{1}-\sigma_{0}}\right) \leq 2^{\max (0,1 / p-1)} K\left(t, f ; L_{p}^{\sigma_{0}}, L_{p}^{\sigma_{1}}\right) .
$$

On the other hand, if $a_{k}=b_{k}+c_{k}$ with $\left\{b_{k}\right\} \in l_{p}$ and $\left\{c_{k}\right\} \in l_{p}^{\sigma_{1}-\sigma_{0}}$, then by putting

$$
f \chi_{\Omega_{k}}=f \chi_{\Omega_{k}} \frac{b_{k}}{a_{k}}+f \chi_{\Omega_{k}} \frac{c_{k}}{a_{k}} \quad \text { when } a_{k} \neq 0
$$

we obtain

$$
\begin{aligned}
K\left(t, f ; L_{p}^{\sigma_{0}}, L_{p}^{\sigma_{1}}\right) & \leq\left\|\sum_{k \in \mathbb{Z}} f \chi_{\Omega_{k}} \frac{b_{k}}{a_{k}}\right\|_{L_{p}^{\sigma_{0}}}+t\left\|\sum_{k \in \mathbb{Z}} f \chi_{\Omega_{k}} \frac{c_{k}}{a_{k}}\right\|_{L_{p}^{\sigma_{1}}} \\
& =\left(\sum_{k \in \mathbb{Z}} \int_{\Omega_{k}}\left(\left|\frac{b_{k}}{a_{k}} f(x) w(x)^{\sigma_{0}}\right|\right)^{p} d \mu\right)^{1 / p}
\end{aligned}
$$




$$
\begin{aligned}
& +t\left(\sum_{k \in \mathbb{Z}} \int_{\Omega_{k}}\left(\left|\frac{c_{k}}{a_{k}} f(x) w(x)^{\sigma_{1}}\right|\right)^{p} d \mu\right)^{1 / p} \\
\leq & \left\|\left\{b_{k}\right\}\right\|_{l_{p}}+t\left(\sum_{k \in \mathbb{Z}} \int_{\Omega_{k}}\left(\left|\frac{c_{k}}{a_{k}} f(x) w(x)^{\sigma_{0}} 2^{(k+1)\left(\sigma_{1}-\sigma_{0}\right)}\right|\right)^{p} d \mu\right)^{1 / p} \\
= & \left\|\left\{b_{k}\right\}\right\|_{l_{p}}+t 2^{\sigma_{1}-\sigma_{0}}\left\|\left\{2^{k\left(\sigma_{1}-\sigma_{0}\right)} c_{k}\right\}\right\|_{l_{p}}
\end{aligned}
$$

or

$$
K\left(t, f ; L_{p}^{\sigma_{0}}, L_{p}^{\sigma_{1}}\right) \leq 2^{\sigma_{1}-\sigma_{0}} K\left(t,\left\{a_{k}\right\} ; l_{p}, l_{p}^{\sigma_{1}-\sigma_{0}}\right) .
$$

The equivalence (3.4) and the well known interpolation formula

$$
\left(l_{p}, l_{p}^{\sigma_{1}-\sigma_{0}}\right)_{\theta, q}=l_{q}^{\theta\left(\sigma_{1}-\sigma_{0}\right)}
$$

(see e.g. [BL], Th. 5.6.1) give

$$
\|f\|_{\left(L_{p}^{\sigma_{0}}, L_{p}^{\sigma_{1}}\right)_{\theta, q}} \approx\left\|\left\{a_{k}\right\}\right\|_{l_{q}^{\theta\left(\sigma_{1}-\sigma_{0}\right)}}
$$

and going back from the sequence $\left\{a_{k}\right\}$ to the function $f$ we obtain

$$
\begin{aligned}
\left\|\left\{a_{k}\right\}\right\|_{l_{q}^{\theta\left(\sigma_{1}-\sigma_{0}\right)}} & =\left(\sum_{k \in \mathbb{Z}}\left(2^{k \theta\left(\sigma_{1}-\sigma_{0}\right)} a_{k}\right)^{q}\right)^{1 / q} \\
& =\left(\sum_{k \in \mathbb{Z}} 2^{k q \theta\left(\sigma_{1}-\sigma_{0}\right)}\left(\int_{\Omega_{k}}\left|f(x) w(x)^{\sigma_{0}}\right|^{p} d \mu\right)^{q / p}\right)^{1 / q} \\
& \geq 2^{\sigma_{0}-\sigma_{1}}\left(\sum_{k \in \mathbb{Z}}\left(\int_{\Omega_{k}}\left|w(x)^{\theta\left(\sigma_{1}-\sigma_{0}\right)} f(x) w(x)^{\sigma_{0}}\right|^{p} d \mu\right)^{q / p}\right)^{1 / q} \\
& =2^{\sigma_{0}-\sigma_{1}}\|f\|_{L_{p}^{\sigma, q}}
\end{aligned}
$$

and, similarly,

$$
\left\|\left\{a_{k}\right\}\right\|_{l_{q}^{\theta\left(\sigma_{1}-\sigma_{0}\right)}} \leq\|f\|_{L_{p}^{\sigma, q}}
$$

which means that

$$
\left(L_{p}^{\sigma_{0}}, L_{p}^{\sigma_{1}}\right)_{\theta, q}=L_{p}^{\sigma, q}
$$

with equivalent norms. The proof is complete.

Next we prove that if $p_{0} \neq p_{1}$, then the spaces $L_{p, r}^{\sigma, q}$ are stable under diagonal interpolation.

LEMMA 3.2. If $p_{0} \neq p_{1}, 0<q_{0}, q_{1}<\infty$ and $1 / q=(1-\theta) / q_{0}+\theta / q_{1}$, then

$$
\left(L_{p_{0}, r_{0}}^{\sigma_{0}, q_{0}}, L_{p_{1}, r_{1}}^{\sigma_{1}, q_{1}}\right)_{\theta, q}=L_{p, q}^{\sigma, q},
$$

where $\sigma=(1-\theta) \sigma_{0}+\theta \sigma_{1}$ and $1 / p=(1-\theta) / p_{0}+\theta / p_{1}$.

Proof. Denote the interpolation space $\left(L_{p_{0}, r_{0}}^{\sigma_{0}, q_{0}}, L_{p_{1}, r_{1}}^{\sigma_{1}, q_{1}}\right)_{\theta, q}$ by $L$ and let $g_{k}(x)=f(x) w(x)^{\sigma_{0}} \chi_{\Omega_{k}}(x), \quad$ where $\Omega_{k}, k \in \mathbb{Z}$, are defined by (3.2). 
Then, as $q_{0}, q_{1}<\infty$, we can use the power theorem (see e.g. [BL], Th. 3.11.6) to obtain

$$
L^{q}=\left(\left(L_{p_{0}, r_{0}}^{\sigma_{0}, q_{0}}\right)^{q_{0}},\left(L_{p_{1}, r_{1}}^{\sigma_{1}, q_{1}}\right)^{q_{1}}\right)_{\eta, 1}, \quad \text { where } \quad \eta=\theta q / q_{1} .
$$

Moreover,

$$
\left(\|f\|_{L_{p_{0}, r_{0}}^{\sigma_{0}, q_{0}}}\right)^{q_{0}}=\sum_{k \in \mathbb{Z}}\left(\left\|f w^{\sigma_{0}} \chi_{\Omega_{k}}\right\|_{L_{p_{0}, r_{0}}}\right)^{q_{0}}=\sum_{k \in \mathbb{Z}}\left(\left\|g_{k}\right\|_{L_{p_{0}, r_{0}}}\right)^{q_{0}}
$$

and, according to (3.3),

$$
\begin{aligned}
\left(\|f\|_{L_{p_{1}, r_{1}}^{\sigma_{1}, q_{1}}}\right)^{q_{1}} & =\sum_{k \in \mathbb{Z}}\left(\left\|f w^{\sigma_{1}} \chi_{\Omega_{k}}\right\|_{L_{p_{1}, r_{1}}}\right)^{q_{1}} \\
& =\sum_{k \in \mathbb{Z}}\left(\left\|w^{\sigma_{1}-\sigma_{0}} f w^{\sigma_{0}} \chi_{\Omega_{k}}\right\|_{L_{p_{1}, r_{1}}}\right)^{q_{1}} \\
& =\sum_{k \in \mathbb{Z}} 2^{k\left(\sigma_{1}-\sigma_{0}\right) q_{1}}\left(\left\|g_{k}\right\|_{L_{p_{1}, r_{1}}}\right)^{q_{1}} .
\end{aligned}
$$

Note that the family $\left\{\Omega_{k}\right\}$ covers $\Omega$ and that $\Omega_{k}$ are not overlapping. Therefore, by using (3.7) and (3.8), we see that

$$
K\left(t, f ;\left(L_{p_{0}, r_{0}}^{\sigma_{0}, q_{0}}\right)^{q_{0}},\left(L_{p_{1}, r_{1}}^{\sigma_{1}, q_{1}}\right)^{q_{1}}\right) \approx \sum_{k \in \mathbb{Z}} K\left(t, g_{k} ;\left(L_{p_{0}, r_{0}}\right)^{q_{0}},\left(2^{k\left(\sigma_{1}-\sigma_{0}\right)} L_{p_{1}, r_{1}}\right)^{q_{1}}\right) .
$$

In view of (3.6) and the additivity of the integral it follows that

$$
\|f\|_{L}^{q} \approx \sum_{k \in \mathbb{Z}}\left\|g_{k}\right\|_{L_{k}}, \text { where } L_{k}:=\left(\left(L_{p_{0}, r_{0}}\right)^{q_{0}},\left(2^{k\left(\sigma_{1}-\sigma_{0}\right)} L_{p_{1}, r_{1}}\right)^{q_{1}}\right)_{\eta, 1} .
$$

By using the power theorem once more we find that

(3.10) $\left(\left(L_{p_{0}, r_{0}}\right)^{q_{0}},\left(2^{k\left(\sigma_{1}-\sigma_{0}\right)} L_{p_{1}, r_{1}}\right)^{q_{1}}\right)_{\eta, 1}=\left(\left(L_{p_{0}, r_{0}}, 2^{k\left(\sigma_{1}-\sigma_{0}\right)} L_{p_{1}, r_{1}}\right)_{\theta, q}\right)^{q}$.

Next we recall that for any quasi-Banach couple,

$$
\|x\|_{\left(X_{0}, c X_{1}\right)_{\theta, q}}=c^{\theta}\|x\|_{\left(X_{0}, X_{1}\right)_{\theta, q}} \text { for any } c>0,
$$

and therefore by the well known result on interpolation of Lorentz spaces for $p_{0} \neq p_{1}$ (see [BL], Th. 5.3.1) we see that

$$
\left(L_{p_{0}, r_{0}}, 2^{k\left(\sigma_{1}-\sigma_{0}\right)} L_{p_{1}, r_{1}}\right)_{\theta, q}=2^{k \theta\left(\sigma_{1}-\sigma_{0}\right)} L_{p, q} .
$$

Hence, by using (3.3), (3.9), (3.10), we find that

$$
\begin{aligned}
\|f\|_{L}^{q} & =\sum_{k \in \mathbb{Z}}\left(\left\|f w^{\sigma_{0}} \chi_{\Omega_{k}}\right\|_{2^{k \theta\left(\sigma_{1}-\sigma_{0}\right)} L_{p, q}}\right)^{q} \\
& \approx \sum_{k \in \mathbb{Z}}\left(\left\|f w^{\sigma_{0}} w^{\theta\left(\sigma_{1}-\sigma_{0}\right)} \chi_{\Omega_{k}}\right\|_{L_{p, q}}\right)^{q} \\
& =\sum_{k \in \mathbb{Z}}\left(\left\|f w^{\sigma} \chi_{\Omega_{k}}\right\|_{L_{p, q}}\right)^{q}=\left(\|f\|_{L_{p, q}^{\sigma, q}}\right)^{q}
\end{aligned}
$$

and (3.5) is proved. 
REMARK 3.3. By analyzing the proof of Lemma 3.2 we find that the formula (3.5) is true also for the case $p_{0}=p_{1}$ if we impose the additional condition

$$
1 / q=(1-\theta) / r_{0}+\theta / r_{1} .
$$

The following example will show that in the off-diagonal case, i.e., when $1 / q \neq(1-\theta) / p_{0}+\theta / p_{1}$, the spaces $\left(L_{p_{0}}^{\sigma_{0}}, L_{p_{1}}^{\sigma_{1}}\right)_{\theta, q}$ do not belong in general to the scale of $L_{p, r}^{\sigma, q}$-spaces. This example will be used later on in the construction of counterexamples.

EXAMPLE 3.4. Let $\Omega$ be the set of natural numbers with the measure $\mu(\{n\})=1$ and let the weight function $w$ be defined by $w(n)=2^{n}$. If $p_{0} \neq p_{1}$ and $\sigma_{0}=\sigma_{1}=\sigma$, then we have the usual weighted Lorentz spaces

$$
\left(L_{p_{0}}^{\sigma}, L_{p_{1}}^{\sigma}\right)_{\theta, q}=L_{p, q}\left(w^{\sigma}\right), \quad 1 / p=(1-\theta) / p_{0}+\theta / p_{1},
$$

and

$$
\|f\|_{L_{p, q}\left(w^{\sigma}\right)}=\left\|\left\{2^{n \sigma} f(n)\right\}\right\|_{l_{p, q}} .
$$

On the other hand, as $\Omega_{k}=\{k\}$,

$$
\begin{aligned}
\|f\|_{L_{p_{0}, r_{0}}^{\sigma_{0}, q_{0}}} & =\left(\sum_{k \in \mathbb{Z}}\left(\left\|f w^{\sigma_{0}} \chi_{\Omega_{k}}\right\|_{L_{p_{0}, r_{0}}}\right)^{q_{0}}\right)^{1 / q_{0}} \\
& =\left(\sum_{k \in \mathbb{N}}\left(\int_{0}^{1}\left[t^{1 / p_{0}}|f(k)| 2^{k \sigma_{0}}\right]^{r_{0}} \frac{d t}{t}\right)^{q_{0} / r_{0}}\right)^{1 / q_{0}} \\
& =c\left(\sum_{k \in \mathbb{N}}\left(|f(k)| 2^{k \sigma_{0}}\right)^{q_{0}}\right)^{1 / q_{0}} .
\end{aligned}
$$

If the norms (3.11) and (3.12) are equivalent, then we must have $\sigma_{0}=\sigma$, and thus the second norm can be written as

$$
\|f\|_{L_{p_{0}, r_{0}}^{\sigma_{0}, q_{0}}}=c\left(\sum_{k \in \mathbb{N}}\left(|f(k)| 2^{k \sigma}\right)^{q_{0}}\right)^{1 / q_{0}} .
$$

Hence, by setting $b_{k}=|f(k)| 2^{k \sigma}$, we find that

$$
\left\|\left\{b_{n}\right\}\right\|_{l_{p, q}} \approx\left\|\left\{b_{n}\right\}\right\|_{l_{q_{0}}},
$$

which is possible only when $p=q=q_{0}$, but this is not the case when $q \neq p$. This contradiction shows that the norms in (3.11) and (3.12) are not equivalent, i.e. $\left(L_{p_{0}}^{\sigma}, L_{p_{1}}^{\sigma}\right)_{\theta, q} \neq L_{p_{0}, r_{0}}^{\sigma_{0}, q_{0}}$.

4. Interpolation of triples of weighted $L_{p}$-spaces. We first recall that it was shown in Example 3.4 that in the off-diagonal case the spaces $\left(L_{p_{0}}^{\sigma_{0}}, L_{p_{1}}^{\sigma_{1}}\right)_{\theta, q}$ do not in general belong to the scale of $L_{p, r}^{\sigma, q}$-spaces. According to the main result of this section (Theorem 4.1), the situation is completely different when we interpolate triples $\left(L_{p_{0}}^{\sigma_{0}}, L_{p_{1}}^{\sigma_{1}}, L_{p_{2}}^{\sigma_{2}}\right)$ of weighted $L_{p^{-}}$-spaces. 
Theorem 4.1. Let $0<q<\infty$. If the points $\left(\sigma_{i}, 1 / p_{i}\right), i=0,1,2$, are not collinear, then

$$
\left(L_{p_{0}}^{\sigma_{0}}, L_{p_{1}}^{\sigma_{1}}, L_{p_{2}}^{\sigma_{2}}\right)_{\left(\theta_{1}, \theta_{2}\right), q}=L_{p, q}^{\sigma, q}
$$

where

$$
(\sigma, 1 / p)=\left(1-\theta_{1}-\theta_{2}\right)\left(\sigma_{0}, 1 / p_{0}\right)+\theta_{1}\left(\sigma_{1}, 1 / p_{1}\right)+\theta_{2}\left(\sigma_{2}, 1 / p_{2}\right) .
$$

The description (4.1) also holds for the case $q=\infty$ if we impose the additional assumption that $p_{0}, p_{1}, p_{2} \geq 1$.

REMARK 4.2. Let us mention that in Theorem 4.1 we have implicitly assumed that $0<p<\infty$ because if $p=\infty$, then $p_{0}=p_{1}=p_{2}=\infty$ and the points $\left(\sigma_{i}, 1 / p_{i}\right), i=0,1,2$, are on the same line, which contradicts the assumption.

REMARK 4.3. In the case when the points $\left(\sigma_{i}, 1 / p_{i}\right), i=0,1,2$, lie on the same line, Theorem 4.1 is not true in general. Let, for example, $p_{1}<p_{2}<p_{0}$. Then there exists $\lambda \in(0,1)$ such that

$$
1 / p_{2}=(1-\lambda) / p_{0}+\lambda / p_{1} \text { and } \sigma_{2}=(1-\lambda) \sigma_{0}+\lambda \sigma_{1},
$$

and from the Stein-Weiss theorem (see [BL], Th. 5.5.1) we have $L_{p_{2}}^{\sigma_{2}}=$ $\left(L_{p_{0}}^{\sigma_{0}}, L_{p_{1}}^{\sigma_{1}}\right)_{\lambda, p_{2}}$. Then, in view of Theorem 9.1 of Sparr [S], we have

$$
\left(L_{p_{0}}^{\sigma_{0}}, L_{p_{1}}^{\sigma_{1}}, L_{p_{2}}^{\sigma_{2}}\right)_{\left(\theta_{1}, \theta_{2}\right), q}=\left(L_{p_{0}}^{\sigma_{0}}, L_{p_{1}}^{\sigma_{1}}\right)_{\mu, q}
$$

with $\mu=\theta_{1}+\lambda \theta_{2}$ and $1 \leq q \leq \infty$. For $0<q<\infty$ this can also be shown from our Corollary 2.6 and the reiteration theorem for couples:

$$
\begin{aligned}
\left(L_{p_{0}}^{\sigma_{0}}, L_{p_{1}}^{\sigma_{1}}, L_{p_{2}}^{\sigma_{2}}\right)_{\left(\theta_{1}, \theta_{2}\right), q} & \\
& =\left(\left(L_{p_{0}}^{\sigma_{0}},\left(L_{p_{0}}^{\sigma_{0}}, L_{p_{1}}^{\sigma_{1}}\right)_{\lambda, p_{2}}\right)_{\theta_{2}, q},\left(L_{p_{1}}^{\sigma_{1}},\left(L_{p_{0}}^{\sigma_{0}}, L_{p_{1}}^{\sigma_{1}}\right)_{\lambda, p_{2}}\right)_{\theta_{2}, q}\right)_{\theta_{1} /\left(1-\theta_{2}\right), q} \\
& =\left(L_{p_{0}}^{\sigma_{0}}, L_{p_{1}}^{\sigma_{1}}\right)_{\mu, q} .
\end{aligned}
$$

In the case when $q$ is non-diagonal, i.e.,

$$
1 / q \neq(1-\mu) / p_{0}+\mu / p_{1}=\left(1-\theta_{1}-\theta_{2}\right) / p_{0}+\theta_{1} / p_{1}+\theta_{2} / p_{2},
$$

the space $\left(L_{p_{0}}^{\sigma_{0}}, L_{p_{1}}^{\sigma_{1}}\right)_{\mu, q}$ is different from $L_{p, q}^{\sigma, q}$ so Theorem 4.1 is not true.

For the proof of Theorem 4.1 we need two lemmas.

LEMmA 4.4. If $q$ is diagonal, i.e., when $1 / q=1 / p=\left(1-\theta_{1}-\theta_{2}\right) / p_{0}+$ $\theta_{1} / p_{1}+\theta_{2} / p_{2}$, then the formula (4.1) holds even without the restriction that the points $\left(\sigma_{i}, 1 / p_{i}\right), i=0,1,2$, are not collinear.

Proof. From Sparr's result [S, Th. 8.1] it follows that the right hand side of (4.1) is equal to $L_{p}^{\sigma}$. Since $q=p$ it follows that $L_{p}^{\sigma}=L_{p, q}^{\sigma, q}$.

In the next lemma we consider two triples $\left(L_{p_{0}}^{\sigma_{0}}, L_{p_{1}}^{\sigma_{1}}, L_{p_{2}}^{\sigma_{2}}\right),\left(L_{r_{0}}^{\alpha_{0}}, L_{r_{1}}^{\alpha_{1}}, L_{r_{2}}^{\alpha_{2}}\right)$ and the set of parameters from $H$ given by (1.1). 
LemMA 4.5. Assume that the points $\left(\sigma_{i}, 1 / p_{i}\right), i=0,1,2$, are not collinear and also the points $\left(\alpha_{i}, 1 / r_{i}\right), i=0,1,2$, are not collinear. If $\left(\theta_{1}, \theta_{2}\right)$, $\left(\lambda_{1}, \lambda_{2}\right) \in H$ and

$$
\begin{aligned}
& \left(1-\theta_{1}-\theta_{2}\right)\left(\sigma_{0}, 1 / p_{0}\right)+\theta_{1}\left(\sigma_{1}, 1 / p_{1}\right)+\theta_{2}\left(\sigma_{2}, 1 / p_{2}\right) \\
& =\left(1-\lambda_{1}-\lambda_{2}\right)\left(\alpha_{0}, 1 / r_{0}\right)+\lambda_{1}\left(\alpha_{1}, 1 / r_{1}\right)+\lambda_{2}\left(\alpha_{2}, 1 / r_{2}\right),
\end{aligned}
$$

then

$$
\left(L_{p_{0}}^{\sigma_{0}}, L_{p_{1}}^{\sigma_{1}}, L_{p_{2}}^{\sigma_{2}}\right)_{\left(\theta_{1}, \theta_{2}\right), q}=\left(L_{r_{0}}^{\alpha_{0}}, L_{r_{1}}^{\alpha_{1}}, L_{r_{2}}^{\alpha_{2}}\right)_{\left(\lambda_{1}, \lambda_{2}\right), q} .
$$

Proof. Denote by $\Delta$ the triangle with vertices $\left(\sigma_{i}, 1 / p_{i}\right)$ and by $\Delta^{\prime}$ the triangle with vertices $\left(\alpha_{i}, 1 / r_{i}\right), i=0,1,2$. Then (4.2) and the assumption $\left(\theta_{1}, \theta_{2}\right),\left(\lambda_{1}, \lambda_{2}\right) \in H$ mean that the point

$$
\begin{aligned}
x^{*} & :=\left(1-\theta_{1}-\theta_{2}\right)\left(\sigma_{0}, 1 / p_{0}\right)+\theta_{1}\left(\sigma_{1}, 1 / p_{1}\right)+\theta_{2}\left(\sigma_{2}, 1 / p_{2}\right) \\
& =\left(1-\lambda_{1}-\lambda_{2}\right)\left(\alpha_{0}, 1 / r_{0}\right)+\lambda_{1}\left(\alpha_{1}, 1 / r_{1}\right)+\lambda_{2}\left(\alpha_{2}, 1 / r_{2}\right)
\end{aligned}
$$

belongs to the interior of both $\Delta$ and $\Delta^{\prime}$. In the interior of $\Delta \cap \Delta^{\prime}$, take three points $\left(\delta_{i}, 1 / q_{i}\right), i=0,1,2$, such that $x^{*}$ belongs to the interior of the triangle with these vertices. Thus, there are strictly positive numbers $\eta_{0}, \eta_{1}$ and $\eta_{2}$ such that $\eta_{0}+\eta_{1}+\eta_{2}=1$ and

$$
x^{*}:=\eta_{0}\left(\delta_{0}, 1 / q_{0}\right)+\eta_{1}\left(\delta_{1}, 1 / q_{1}\right)+\eta_{2}\left(\delta_{2}, 1 / q_{2}\right) .
$$

Moreover, since the points $\left(\delta_{i}, 1 / q_{i}\right)$ belong to the interior of both $\Delta$ and $\Delta^{\prime}$, there exist vectors $\left(\theta_{1}^{i}, \theta_{2}^{i}\right),\left(\lambda_{1}^{i}, \lambda_{2}^{i}\right) \in H, i=0,1,2$, such that

$$
\begin{aligned}
\left(\delta_{i}, 1 / q_{i}\right) & =\left(1-\theta_{1}^{i}-\theta_{2}^{i}\right)\left(\sigma_{0}, 1 / p_{0}\right)+\theta_{1}^{i}\left(\sigma_{1}, 1 / p_{1}\right)+\theta_{2}^{i}\left(\sigma_{2}, 1 / p_{2}\right) \\
& =\left(1-\lambda_{1}^{i}-\lambda_{2}^{i}\right)\left(\alpha_{0}, 1 / r_{0}\right)+\lambda_{1}^{i}\left(\alpha_{1}, 1 / r_{1}\right)+\lambda_{2}^{i}\left(\alpha_{2}, 1 / r_{2}\right), \quad i=0,1,2 .
\end{aligned}
$$

According to our reiteration Theorem 2.1 we have

$$
\left(L_{p_{0}}^{\sigma_{0}}, L_{p_{1}}^{\sigma_{1}}, L_{p_{2}}^{\sigma_{2}}\right)_{\left(\theta_{1}, \theta_{2}\right), q}=\left(X_{0}, X_{1}, X_{2}\right)_{\left(\eta_{1}, \eta_{2}\right), q},
$$

where

$$
X_{i}=\left(L_{p_{0}}^{\sigma_{0}}, L_{p_{1}}^{\sigma_{1}}, L_{p_{2}}^{\sigma_{2}}\right)_{\left(\theta_{1}^{i}, \theta_{2}^{i}\right), q_{i}}, \quad i=0,1,2,
$$

and also

$$
\left(L_{r_{0}}^{\alpha_{0}}, L_{r_{1}}^{\alpha_{1}}, L_{r_{2}}^{\alpha_{2}}\right)_{\left(\theta_{1}, \theta_{2}\right), q}=\left(Y_{0}, Y_{1}, Y_{2}\right)_{\left(\eta_{1}, \eta_{2}\right), q},
$$

where

$$
Y_{i}=\left(L_{r_{0}}^{\alpha_{0}}, L_{r_{1}}^{\alpha_{1}}, L_{r_{2}}^{\alpha_{2}}\right)_{\left(\lambda_{1}^{i}, \lambda_{2}^{i}\right), q_{i}}, \quad i=0,1,2 .
$$

The desired relation (4.3) follows by comparing (4.4) with (4.5) and using our Lemma 4.4 to conclude that $X_{i}=Y_{i}=L_{q_{i}}^{\delta_{i}}=L_{q_{i}, q_{i}}^{\delta_{i}, q_{i}}, i=0,1,2$. The proof is complete.

We are now ready to present the proof of Theorem 4.1.

Proof of Theorem 4.1. (a) The case $q<\infty$. When $q=p$ the result holds according to Lemma 4.4 so we assume that $q \neq p$. 
From Lemma 4.5 it follows that it is sufficient to find points $\left(\alpha_{i}, 1 / r_{i}\right)$, $i=0,1,2$, such that

$$
(\sigma, 1 / p)=\left(1-\lambda_{1}-\lambda_{2}\right)\left(\alpha_{0}, 1 / r_{0}\right)+\lambda_{1}\left(\alpha_{1}, 1 / r_{1}\right)+\lambda_{2}\left(\alpha_{2}, 1 / r_{2}\right)
$$

and for which

$$
\left(L_{r_{0}}^{\alpha_{0}}, L_{r_{1}}^{\alpha_{1}}, L_{r_{2}}^{\alpha_{2}}\right)_{\left(\lambda_{1}, \lambda_{2}\right), q}=L_{p, q}^{\sigma, q}
$$

We construct them in the following way: first take $M=(\sigma / 2,1 / q)$ (see Figure 3). Since $q<\infty$ we can find points $\left(\alpha_{0}, 1 / r_{0}\right)$ and $\left(\alpha_{2}, 1 / r_{2}\right)$ such that $\alpha_{0}=\alpha_{2}=\sigma / 2$ and as $0<p<\infty$ (cf. Remark 4.2),

$$
1 / r_{0}<\min (1 / p, 1 / q) \leq \max (1 / p, 1 / q)<1 / r_{2} .
$$

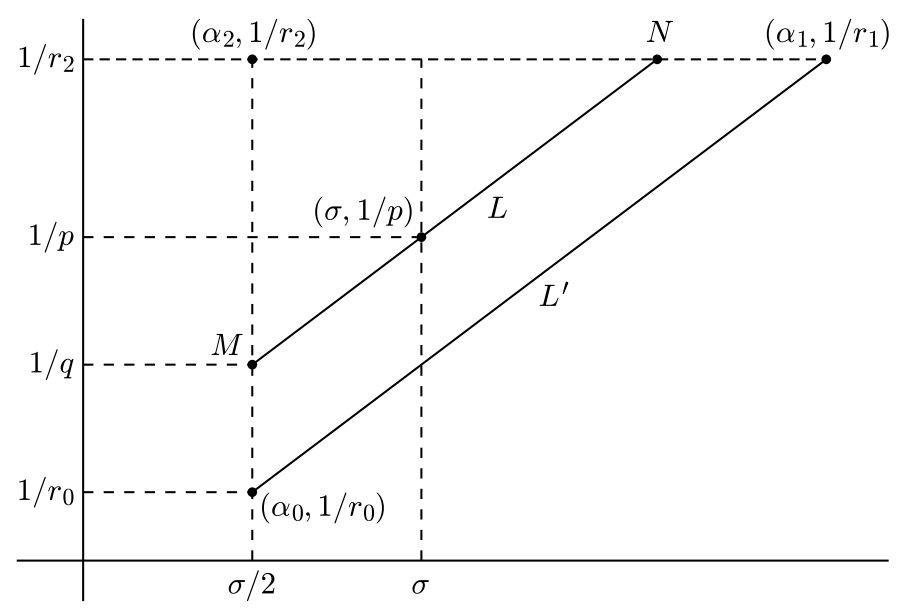

Fig. 3

Then, according to (3.1) we have

$$
\left(L_{r_{0}}^{\alpha_{0}}, L_{r_{2}}^{\alpha_{2}}\right)_{\lambda, q}=\left(L_{r_{0}}^{\sigma / 2}, L_{r_{2}}^{\sigma / 2}\right)_{\lambda, q}=L_{q}^{\sigma / 2}
$$

provided

$$
(1-\lambda) / r_{0}+\lambda / r_{2}=1 / q, \quad \text { i.e., } \quad \lambda=\left(1 / q-1 / r_{0}\right) /\left(1 / r_{2}-1 / r_{0}\right) .
$$

Now consider the straight line $L$ through $M$ and $(\sigma, 1 / p)$. Moreover, we denote by $N$ the point of intersection of $L$ and the horizontal line through $\left(1 / r_{2}, \sigma / 2\right)$ if $1 / p>1 / q$, and through $\left(1 / r_{0}, \sigma / 2\right)$ if $1 / p<1 / q$ (see Figure 3$)$.

It is sufficient to consider the first case because the second can be dealt with in an analogous way. The first coordinate $\sigma^{*}$ of the point $N=\left(\sigma^{*}, 1 / r_{2}\right)$ can easily be calculated by using the obvious symmetry of triangles:

$$
\frac{\sigma^{*}-\sigma}{\sigma^{*}-\sigma / 2}=\frac{1 / r_{2}-1 / p}{1 / r_{2}-1 / q}
$$


We also consider the line $L^{\prime}$, parallel to $L$ and passing through $\left(\sigma / 2,1 / r_{0}\right)$. The intersection of $L^{\prime}$ and the horizontal line through $\left(\sigma / 2,1 / r_{2}\right)$ and $N$ will be our third required point $\left(\alpha_{1}, 1 / r_{1}\right)$, and so we have

$$
1 / r_{1}=1 / r_{2} \text { and }(1-\lambda) \alpha_{1}+\lambda \sigma / 2=\sigma^{*}
$$

with the same $\lambda$ as in (4.9) (because $L^{\prime}$ is parallel to $L$ ). Since $r_{1}=r_{2}$ it follows from Lemma 3.1 and (4.11) that

$$
\left(L_{r_{1}}^{\alpha_{1}}, L_{r_{2}}^{\alpha_{2}}\right)_{\lambda, q}=\left(L_{r_{2}}^{\alpha_{1}}, L_{r_{2}}^{\sigma / 2}\right)_{\lambda, q}=L_{r_{2}}^{(1-\lambda) \alpha_{1}+\lambda \sigma / 2, q}=L_{r_{2}}^{\sigma^{*}, q},
$$

where $\sigma^{*}$ is the first coordinate of $N$ (see (4.10)).

If we take $\eta$ such that

$$
(1-\eta) \sigma / 2+\eta \sigma^{*}=\sigma,
$$

then as the points $(\sigma / 2,1 / q),(\sigma, 1 / p)$ and $\left(\sigma^{*}, 1 / r_{2}\right)$ lie on the same line $L$ we have

$$
(1-\eta) / q+\eta / r_{2}=1 / p
$$

and Lemma 3.2 shows that

$$
\left(L_{q}^{\sigma / 2}, L_{r_{2}}^{\sigma^{*}, q}\right)_{\eta, q}=L_{p, q}^{\sigma, q} .
$$

Thus, according to (4.13), (4.8) and (4.12), we have

$$
L_{p, q}^{\sigma, q}=\left(L_{q}^{\sigma / 2}, L_{r_{2}, q}^{\sigma^{*}, q}\right)_{\eta, q}=\left(\left(L_{r_{0}}^{\alpha_{0}}, L_{r_{2}}^{\alpha_{2}}\right)_{\lambda, q},\left(L_{r_{2}}^{\alpha_{1}}, L_{r_{2}}^{\sigma / 2}\right)_{\lambda, q}\right)_{\eta, q} .
$$

We now put $\lambda_{1}=(1-\lambda) \eta$ and $\lambda_{2}=\lambda$ and use (4.14) and our Corollary 2.6 to conclude that

$$
L_{p, q}^{\sigma, q}=\left(L_{r_{0}}^{\alpha_{0}}, L_{r_{1}}^{\alpha_{1}}, L_{r_{2}}^{\alpha_{2}}\right)_{\left(\lambda_{1}, \lambda_{2}\right), q},
$$

and thus, according to Lemma 4.5, we see that (4.1) holds and the proof of the case $q<\infty$ is complete.

(b) The case $q=\infty$ with the additional assumption $p_{0}, p_{1}, p_{2}>1$. In this case we can use the well known duality

$$
\left(L_{q_{i}}^{-\sigma_{i}}\right)^{*}=L_{p_{i}}^{\sigma_{i}}, \quad 1 / q_{i}+1 / p_{i}=1, i=0,1,2 .
$$

For $q=1$ we have proved above that

$$
\left(L_{q_{0}}^{-\sigma_{0}}, L_{q_{1}}^{-\sigma_{1}}, L_{q_{2}}^{-\sigma_{2}}\right)_{\left(\theta_{1}, \theta_{2}\right), 1}=L_{q, 1}^{-\sigma, 1},
$$

where $(\sigma, 1 / q)=\left(1-\theta_{0}-\theta_{1}\right)\left(\sigma_{0}, 1 / q_{0}\right)+\theta_{1}\left(\sigma_{1}, 1 / q_{1}\right)+\theta_{2}\left(\sigma_{2}, 1 / q_{2}\right)$. Therefore by using the duality of $K$ - and $J$-methods (see [CFMR], Th. 3.4) and the equivalence of $K$ - and $J$-methods for Banach function lattices on $\Omega$ (see [AK], Th. 1) we obtain

$$
\left(L_{p_{0}}^{\sigma_{0}}, L_{p_{1}}^{\sigma_{1}}, L_{p_{2}}^{\sigma_{2}}\right)_{\left(\theta_{1}, \theta_{2}\right), \infty}=\left(\left(L_{q_{0}}^{-\sigma_{0}}, L_{q_{1}}^{-\sigma_{1}}, L_{q_{2}}^{-\sigma_{2}}\right)_{\left(\theta_{1}, \theta_{2}\right), 1}\right)^{*}=\left(L_{q, 1}^{-\sigma, 1}\right)^{*} .
$$

It only remains to prove that

$$
\left(L_{q, 1}^{-\sigma, 1}\right)^{*}=L_{p, \infty}^{\sigma, \infty}, \quad 1 / p+1 / q=1
$$


but this formula follows at once from the well known duality of Lorentz spaces $\left[L_{q, 1}\left(w^{-\sigma}\right)\right]^{*}=L_{p, \infty}\left(w^{\sigma}\right)$ and the definition of the spaces $L_{q, 1}^{-\sigma, 1}$. Indeed, $L_{q, 1}^{-\sigma, 1}=l_{1}\left[L_{q, 1}\left(w^{-\sigma}\right)\right]$ in the sense that

$$
\|f\|_{L_{q, 1}^{-\sigma, 1}}=\sum_{k \in \mathbb{Z}}\left\|f w^{-\sigma} \chi_{\Omega_{k}}\right\|_{L_{q, 1}}=\sum_{k \in \mathbb{Z}}\left\|a_{k}\right\|_{L_{q, 1}\left(w^{-\sigma}\right)}, \quad a_{k}=f \chi_{\Omega_{k}} .
$$

The sets $\Omega_{k}$ do not intersect and therefore

$$
\left(L_{q, 1}^{-\sigma, 1}\right)^{*}=\left\{l_{1}\left[L_{q, 1}\left(w^{-\sigma}\right)\right]\right\}^{*}=l_{\infty}\left[\left\{L_{q, 1}\left(w^{-\sigma}\right)\right\}^{*}\right]=l_{\infty}\left[L_{p, \infty}\left(w^{\sigma}\right)\right]=L_{p, \infty}^{\sigma, \infty} .
$$

(c) The case where $q=\infty$ and at least one of $p_{0}, p_{1}$ and $p_{2}$ is equal to 1 . The case $p_{0}=p_{1}=p_{2}=1$ is not possible since our points are then on one line. In the case when some $p_{i}>1(i=0,1,2)$ we consider the triangle $\Delta$ with vertices $\left(\sigma_{i}, 1 / p_{i}\right)$ and take the points $\left(\alpha_{i}, 1 / r_{i}\right)$ in its interior. Then from Lemma 4.5 it follows that it is enough to calculate the interpolation spaces for the triple $\left(L_{r_{0}}^{\alpha_{0}}, L_{r_{1}}^{\alpha_{1}}, L_{r_{2}}^{\alpha_{2}}\right)$ with $r_{i}>1, i=0,1,2$, which was already done before.

We finish this part by using Theorem 4.1 for calculations which will show that the restrictions in the Second Reiteration Theorem are essential (cf. Remark 2.5).

ExAmple 4.6. Let $X_{0}=L_{p_{0}}^{\sigma_{0}}, X_{1}=L_{p_{1}}^{\sigma_{1}}$ and $X_{2}=L_{p_{0}}^{\sigma_{2}}$ with $\sigma_{1}=$ $\left(1-\alpha_{0}\right) \sigma_{0}+\alpha_{0} \sigma_{2}$. Then

$$
\left(X_{0}, X_{2}\right)_{\alpha_{0}, p_{0}}=\left(L_{p_{0}}^{\sigma_{0}}, L_{p_{0}}^{\sigma_{2}}\right)_{\alpha_{0}, p_{0}}=L_{p_{0}}^{\sigma_{1}}
$$

and so

$$
\left(\left(X_{0}, X_{2}\right)_{\alpha_{0}, p_{0}}, X_{1}\right)_{\mu, q}=\left(L_{p_{0}}^{\sigma_{1}}, L_{p_{1}}^{\sigma_{1}}\right)_{\mu, q}=L_{p, q}\left(w^{\sigma_{1}}\right)
$$

with $1 / p=(1-\mu) / p_{0}+\mu / p_{1}$. On the other hand, from Theorem 4.1 it follows that

$$
\left(X_{0}, X_{1}, X_{2}\right)_{\left(\mu, \alpha_{0}(1-\mu)\right), q}=\left(L_{p_{0}}^{\sigma_{0}}, L_{p_{1}}^{\sigma_{1}}, L_{p_{0}}^{\sigma_{2}}\right)_{\left(\mu, \alpha_{0}(1-\mu)\right), q}=L_{p, q}^{\sigma_{1}, q},
$$

but the last block-Lorentz space $L_{p, q}^{\sigma_{1}, q}$ is different in general from the weighted Lorentz spaces $L_{p, q}\left(w^{\sigma_{1}}\right)$ (cf. Example 3.4) and so

$$
\left(\left(X_{0}, X_{2}\right)_{\alpha_{0}, p_{0}}, X_{1}\right)_{\mu, q} \neq\left(X_{0}, X_{1}, X_{2}\right)_{\left(\mu, \alpha_{0}(1-\mu)\right), q} .
$$

5. Wavelets and stability of smooth function spaces. It is known that the norms in the Besov spaces $B_{p}^{\sigma}$ can be expressed in terms of the wavelet coefficients of the function (see $[\mathrm{Me}],[\mathrm{Ky}],[\mathrm{JM}]$ ). For simplicity we first consider the case when the spaces considered are homogeneous and we denote them by $\dot{B}_{p}^{\sigma}$. 
Let $\left\{\Psi_{I}^{i}\right\}$ ( $I$ is a dyadic cube in $\mathbb{R}^{d}, i=1, \ldots, 2^{d-1}$ ) be an orthonormal wavelet basis. Then (see [HJLY], p. 431)

$$
\|f\|_{\dot{B}_{p}^{\sigma}} \approx\left(\sum_{i, I}\left(\frac{\left\langle f, \Psi_{I}^{i}\right\rangle}{|I|^{\sigma / d+1 / 2-1 / p}}\right)^{p}\right)^{1 / p}
$$

where $|I|$ denotes the volume of $I$ and $\left\langle f, \Psi_{I}^{i}\right\rangle$ are the wavelet coefficients of $f$. For our purposes it is useful to rewrite this representation as

$$
\|f\|_{\dot{B}_{p}^{\sigma}} \approx\left(\sum_{i, I}\left(\frac{\left\langle f, \Psi_{I}^{i}\right\rangle|I|^{-\sigma / d}}{|I|^{1 / 2}}\right)^{p}|I|\right)^{1 / p} .
$$

Moreover, we denote by $\Omega$ the discrete set of pairs $(I, i)$ and introduce a measure $\mu$ on it by putting $\mu(I, i)=|I|$. We also consider the weight $w: \Omega \rightarrow \mathbb{R}_{+}$given by $w(I, i)=|I|^{-1 / d}$. Thus we have

$$
\|f\|_{\dot{B}_{p}^{\sigma}} \approx\left(\sum_{i, I}\left(\frac{\left\langle f, \Psi_{I}^{i}\right\rangle}{|I|^{1 / 2}} w(I, i)^{\sigma}\right)^{p} d \mu(I, i)\right)^{1 / p} .
$$

Therefore the operator $S$ defined by

$$
S f(I, i)=\left\langle f, \Psi_{I}^{i}\right\rangle|I|^{-1 / 2}
$$

is a linear isomorphism between the spaces $\dot{B}_{p}^{\sigma}$ and $L_{p}^{\sigma}=L_{p}\left(\Omega, w^{\sigma} d \mu\right)$ on $\Omega$ with $\mu(I, i)=|I|$ and the weight $w(I, i)=|I|^{-1 / d}$. We remark that a similar representation also holds in the non-homogeneous case, only the set $\Omega$ must be a little different (see [Me]). We denote this set by $\Omega^{\prime}$ and the corresponding operator by $S^{\prime}$. Thus $S^{\prime}$ is a linear isomorphism between $B_{p}^{\sigma}$ and $L_{p}^{\sigma}$ on $\Omega^{\prime}$.

Definition 5.1. Let $X$ be a Banach or quasi-Banach space on $\mathbb{R}^{d}$. We say that $X$ has smoothness index $\sigma$ and integration exponent $p(\sigma \in \mathbb{R}, 0<$ $p \leq \infty)$ if we have continuous imbeddings

$$
B_{p, 1}^{\sigma, 1} \subset X \subset B_{p, \infty}^{\sigma, \infty} .
$$

The class of all such spaces will be denoted $C(\sigma, p)$.

Here and in what follows, $B_{p, r}^{\sigma, q}:=B^{\sigma, q}\left(L_{p, r}\right)$ is a generalized Besov space based on the Lorentz spaces $L_{p, r}$ (which Peetre even calls the Lorentz-Besov spaces - cf. [P], pp. 57-58).

We note that the Besov spaces $B_{p}^{\sigma, q}$ and the Sobolev spaces $W_{p}^{k}$ belong to the classes $C(\sigma, p)$ and $C(k, p)$, respectively, since

$$
B_{p, 1}^{\sigma, 1} \subset B_{p}^{\sigma, 1} \subset B_{p}^{\sigma, q} \subset B_{p}^{\sigma, \infty} \subset B_{p, \infty}^{\sigma, \infty}
$$

and

$$
B_{p, 1}^{k, 1} \subset B_{p}^{k, 1} \subset W_{p}^{k} \subset B_{p}^{k, \infty} \subset B_{p, \infty}^{k, \infty} .
$$

The main result of this section reads: 
THEOREM 5.2. Let $X_{i}$ be Banach or quasi-Banach function spaces on $\mathbb{R}^{d}$ such that $X_{i} \in C\left(\sigma_{i}, p_{i}\right), i=0,1,2$, and assume that the points $\left(\sigma_{i}, 1 / p_{i}\right)$, $i=0,1,2$, are not collinear. If $0<q \leq \infty$ and $1<p_{0}, p_{1}, p_{2}<\infty$, then

$$
\left(X_{0}, X_{1}, X_{2}\right)_{\left(\theta_{1}, \theta_{2}\right), q}=B_{p, q}^{\sigma, q}
$$

where

$$
(\sigma, 1 / p)=\left(1-\theta_{1}-\theta_{2}\right)\left(\sigma_{0}, 1 / p_{0}\right)+\theta_{1}\left(\sigma_{1}, 1 / p_{1}\right)+\theta_{2}\left(\sigma_{2}, 1 / p_{2}\right) .
$$

REMARK 5.3. The assumption that the points $\left(\sigma_{i}, 1 / p_{i}\right), i=0,1,2$, do not lie on the same line is essential. Indeed, from Remark 4.3 and the fact that $S^{\prime}$ is an isomorphism between $B_{p}^{\sigma}$ and $L_{p}^{\sigma}$ it follows that in this case the space $\left(B_{p_{0}}^{\sigma_{0}}, B_{p_{1}}^{\sigma_{1}}, B_{p_{2}}^{\sigma_{2}}\right)_{\left(\theta_{1}, \theta_{2}\right), q}$ coincides with some space of the form $\left(B_{q_{0}}^{\delta_{0}}, B_{q_{1}}^{\delta_{1}}\right)_{\mu, q}$ and it is known that for $q_{0} \neq q_{1}$ and $q$ non-diagonal this space is not in the scale of Besov spaces.

The proof of Theorem 5.2 is based on the following proposition.

Proposition 5.4. Assume that the points $\left(\sigma_{i}, 1 / p_{i}\right), i=0,1,2$, are not collinear. If $0<q \leq \infty$ and $1 \leq p_{0}, p_{1}, p_{2} \leq \infty$, then

$$
\left(B_{p_{0}}^{\sigma_{0}}, B_{p_{1}}^{\sigma_{1}}, B_{p_{2}}^{\sigma_{2}}\right)_{\left(\theta_{1}, \theta_{2}\right), q}=B_{p, q}^{\sigma, q},
$$

where

$$
(\sigma, 1 / p)=\left(1-\theta_{1}-\theta_{2}\right)\left(\sigma_{0}, 1 / p_{0}\right)+\theta_{1}\left(\sigma_{1}, 1 / p_{1}\right)+\theta_{2}\left(\sigma_{2}, 1 / p_{2}\right) .
$$

Proof. Since $S^{\prime}$ is a linear isomorphism between $B_{p}^{\sigma}$ and $L_{p}^{\sigma}$ it follows that $S^{\prime}$ is also a linear isomorphism between $\left(B_{p_{0}}^{\sigma_{0}}, B_{p_{1}}^{\sigma_{1}}, B_{p_{2}}^{\sigma_{2}}\right)_{\left(\theta_{1}, \theta_{2}\right), q}$ and $\left(L_{p_{0}}^{\sigma_{0}}, L_{p_{1}}^{\sigma_{1}}, L_{p_{2}}^{\sigma_{2}}\right)_{\left(\theta_{1}, \theta_{2}\right), q}$. Moreover, according to Theorem 4.1, the last space coincide with $L_{p, q}^{\sigma, q}$, and thus it remains to prove that $S^{\prime}$ is a linear isomorphism between $B_{p, q}^{\sigma, q}$ and $L_{p, q}^{\sigma, q}$.

First of all note that from the assumptions $1 / p=\left(1-\theta_{1}-\theta_{2}\right) / p_{0}+$ $\theta_{1} / p_{1}+\theta_{2} / p_{2}$ and $1 \leq p_{0}, p_{1}, p_{2} \leq \infty$ it follows that $1 \leq p \leq \infty$. Moreover, since the points $\left(\sigma_{i}, 1 / p_{i}\right), i=0,1,2$, do not lie on one straight line, not all $p_{i}$ are 1 and not all $p_{i}$ are $\infty$, and it follows that $1<p<\infty$.

Let us now prove that $S^{\prime}$ is a linear isomorphism between $B_{p, q}^{\sigma, q}$ and $L_{p, q}^{\sigma, q}$. We first note that from the well known equality (see [BL], Th. 6.4.5, (1))

$$
\left(B_{r}^{\alpha_{0}}, B_{r}^{\alpha_{1}}\right)_{\theta, q}=B_{r}^{\alpha, q}, \quad \alpha=(1-\theta) \alpha_{0}+\theta \alpha_{1},
$$

and Lemma 3.1:

$$
\left(L_{r}^{\alpha_{0}}, L_{r}^{\alpha_{1}}\right)_{\theta, q}=L_{r}^{\alpha, q}, \quad \alpha=(1-\theta) \alpha_{0}+\theta \alpha_{1},
$$

it follows that $S^{\prime}$ is an isomorphism between $B_{r}^{\alpha, q}$ and $L_{r}^{\alpha, q}$.

Now, we choose $\beta_{0}, \beta_{1}, r \geq 1$ and $\eta \in(0,1)$ such that

$$
(1-\eta) \beta_{0}+\eta \beta_{1}=\sigma \quad \text { and } \quad(1-\eta) / q+\eta / r=1 / p
$$


(the last equality is possible because $1<p<\infty$ ). Then, according to a result of Peetre (see $[\mathrm{P}]$, Th. 6, p. 106, equality (6))

$$
\left(B_{q}^{\beta_{0}}, B_{r}^{\beta_{1}, q}\right)_{\eta, q}=B_{p, q}^{\sigma, q},
$$

and since, in view of Lemma 3.2 ,

$$
\left(L_{q}^{\beta_{0}}, L_{r}^{\beta_{1}, q}\right)_{\eta, q}=L_{p, q}^{\sigma, q},
$$

it follows that $S^{\prime}$ is an isomorphism between $B_{p, q}^{\sigma, q}$ and $L_{p, q}^{\sigma, q}$. The proof is complete.

Proof of Theorem 5.2. Consider the triangle $\Delta$ with vertices $\left(\sigma_{i}, 1 / p_{i}\right)$, $i=0,1,2$. Since $1<p_{0}, p_{1}, p_{2}<\infty$ it follows that there exists a triangle $\Delta^{\prime}$ with vertices $\left(\alpha_{i}, 1 / r_{i}\right), i=0,1,2$, and $1<r_{0}, r_{1}, r_{2}<\infty$ such that $\Delta$ lies in the interior of $\Delta^{\prime}$. Thus Proposition 5.4 yields

$$
B_{p_{i}, q_{i}}^{\sigma_{i}, q_{i}}=\left(B_{r_{0}}^{\alpha_{0}}, B_{r_{1}}^{\alpha_{1}}, B_{r_{2}}^{\alpha_{2}}\right)_{\left(\lambda_{1}^{i}, \lambda_{2}^{i}\right), q_{i}}, \quad B_{p_{i}}^{\sigma_{i}}=\left(B_{r_{0}}^{\alpha_{0}}, B_{r_{1}}^{\alpha_{1}}, B_{r_{2}}^{\alpha_{2}}\right)_{\left(\lambda_{1}^{i}, \lambda_{2}^{i}\right), p_{i}}
$$

for some parameters $\left(\lambda_{1}^{i}, \lambda_{2}^{i}\right) \in H, i=0,1,2$. Therefore, by the First Reiteration Theorem, we have

$$
\left(B_{p_{0}, 1}^{\sigma_{0}, 1}, B_{p_{1}, 1}^{\sigma_{1}, 1}, B_{p_{2}, 1}^{\sigma_{2}, 1}\right)_{\left(\theta_{1}, \theta_{2}\right), q}=\left(B_{p_{0}}^{\sigma_{0}}, B_{p_{1}}^{\sigma_{1}}, B_{p_{2}}^{\sigma_{2}}\right)_{\left(\theta_{1}, \theta_{2}\right), q}=B_{p, q}^{\sigma, q}
$$

and

$$
\left(B_{p_{0}, \infty}^{\sigma_{0}, \infty}, B_{p_{1}, \infty}^{\sigma_{1}, \infty}, B_{p_{2}, \infty}^{\sigma_{2}, \infty}\right)_{\left(\theta_{1}, \theta_{2}\right), q}=\left(B_{p_{0}}^{\sigma_{0}}, B_{p_{1}}^{\sigma_{1}}, B_{p_{2}}^{\sigma_{2}}\right)_{\left(\theta_{1}, \theta_{2}\right), q}=B_{p, q}^{\sigma, q} .
$$

Since for the spaces $X_{i}$ we have the imbeddings

$$
B_{p_{i}, 1}^{\sigma_{i}, 1} \subset X_{i} \subset B_{p_{i}, \infty}^{\sigma_{i}, \infty}, \quad i=0,1,2,
$$

the above equalities imply (5.2) and the proof is complete.

EXAmple 5.5. Assume that the points $\left(\sigma_{i}, 1 / p_{i}\right), i=0,1,2$, are not collinear. If $0<q \leq \infty$ and $1<p_{0}, p_{1}, p_{2}<\infty$, then

$$
\left(B_{p_{0}}^{\sigma_{0}, q_{0}}, B_{p_{1}}^{\sigma_{1}, q_{1}}, B_{p_{2}}^{\sigma_{2}, q_{2}}\right)_{\left(\theta_{1}, \theta_{2}\right), q}=B_{p, q}^{\sigma, q}
$$

and

$$
\left(W_{p_{0}}^{k_{0}}, W_{p_{1}}^{k_{1}}, W_{p_{2}}^{k_{2}}\right)_{\left(\theta_{1}, \theta_{2}\right), q}=B_{p, q}^{\sigma, q}
$$

where $1 / p=\left(1-\theta_{1}-\theta_{2}\right) / p_{0}+\theta_{1} / p_{1}+\theta_{2} / p_{2}$ and $\sigma=\left(1-\theta_{1}-\theta_{2}\right) \sigma_{0}+$ $\theta_{1} \sigma_{1}+\theta_{2} \sigma_{2}$ or $\sigma=\left(1-\theta_{0}-\theta_{1}\right) k_{0}+\theta_{1} k_{1}+\theta_{2} k_{2}$, respectively.

REMARK 5.6. It would be interesting, and seems possible, to extend Theorem 5.2 to the whole range $0<p_{0}, p_{1}, p_{2} \leq \infty$.

6. Stein-Weiss interpolation theorem for Lorentz spaces with change of measures. In 1958 Stein and Weiss [SW] proved a very useful interpolation theorem, which can be written (see also [BL], Th. 5.4.1 and 5.5.1) as

$$
\left(L_{p_{0}}\left(w_{0} d \mu\right), L_{p_{1}}\left(w_{1} d \mu\right)\right)_{\theta, p}=L_{p}(w d \mu)
$$


where $0<p_{0}, p_{1}<\infty, 0<\theta<1$ and

$$
1 / p=(1-\theta) / p_{0}+\theta / p_{1} \text { and } \quad w=w_{0}^{(1-\theta) p / p_{0}} w_{1}^{\theta p / p_{1}} .
$$

In this connection it is natural to ask for an analogue of formula (6.1) if we replace $L_{p}$-spaces by Lorentz $L_{p, q}$-spaces. However the "natural" formula

$$
\begin{gathered}
\left(L_{p_{0}, q_{0}}\left(w_{0} d \mu\right), L_{p_{1}, q_{1}}\left(w_{1} d \mu\right)\right)_{\theta, q}=L_{p, q}(w d \mu), \\
1 / q=(1-\theta) / q_{0}+\theta / q_{1},
\end{gathered}
$$

is not true in general (cf. Example 6.3).

We are interested in describing the space

$$
L=\left(L_{p_{0}, q_{0}}\left(w_{0} d \mu\right), L_{p_{1}, q_{1}}\left(w_{1} d \mu\right)\right)_{\theta, q} .
$$

We will obtain the description as a corollary of a more general result. Let $w$ be a weight function on $\Omega$ and the sets $\Omega_{k}$ be defined by

$$
\Omega_{k}=\Omega_{k}(w)=\left\{x \in \Omega: 2^{k} \leq w(x)<2^{k+1}\right\}, \quad k \in \mathbb{Z},
$$

and let the Lorentz spaces $L_{p, q}\left(w^{\alpha}, w^{\delta} d \mu\right)$ be given as in the Preliminaries with $\alpha$ and $\delta$ some fixed real numbers.

The main result of this part is the theorem below in which we will need the following conditions:

$$
\begin{aligned}
& \delta_{0} \neq \delta_{1}, \quad \frac{\alpha_{0}-\alpha_{1}}{\delta_{0}-\delta_{1}} \leq 0, \quad \frac{\alpha_{0}-\alpha_{1}}{\delta_{0}-\delta_{1}} \neq-\frac{1}{p_{0}}, \quad \frac{\alpha_{0}-\alpha_{1}}{\delta_{0}-\delta_{1}} \neq-\frac{1}{p_{1}}, \\
& 1 / p=(1-\theta) / p_{0}+\theta / p_{1}, \quad 1 / q=(1-\theta) / q_{0}+\theta / q_{1}, \\
& \alpha=(1-\theta) \alpha_{0}+\theta \alpha_{1}, \quad \delta=(1-\theta) \delta_{0} p / p_{0}+\theta \delta_{1} p / p_{1} .
\end{aligned}
$$

Theorem 6.1. Let $0<p_{0}, p_{1}, q_{0}, q_{1}<\infty, \alpha_{0}, \alpha_{1}, \delta_{0}, \delta_{1} \in \mathbb{R}$ and assume that the conditions (6.5)-(6.7) are satisfied. Then the norm of the interpolation space

$$
L=\left(L_{p_{0}, q_{0}}\left(w^{\alpha_{0}}, w^{\delta_{0}} d \mu\right), L_{p_{1}, q_{1}}\left(w^{\alpha_{1}}, w^{\delta_{1}} d \mu\right)\right)_{\theta, q}
$$

satisfies

$$
\|f\|_{L} \approx\left(\sum_{k \in \mathbb{Z}}\left(\left\|f \chi_{\Omega_{k}}\right\|_{L_{p, q}\left(w^{\alpha}, w^{\delta} d \mu\right)}\right)^{q}\right)^{1 / q},
$$

where the sets $\Omega_{k}$ are given by (6.4).

COROLlary 6.2 (Stein-Weiss interpolation theorem for Lorentz spaces). Let $0<p_{0}, p_{1}, q_{0}, q_{1}<\infty$. Then the interpolation space

$$
L=\left(L_{p_{0}, q_{0}}\left(w_{0} d \mu\right), L_{p_{1}, q_{1}}\left(w_{1} d \mu\right)\right)_{\theta, q}
$$

is a block-Lorentz space with norm

$$
\|f\|_{L} \approx\left(\sum_{k \in \mathbb{Z}}\left(\left\|f \chi_{\Omega_{k}}\right\|_{L_{p, q}(w d \mu)}\right)^{q}\right)^{1 / q},
$$


where

$$
\Omega_{k}=\Omega_{k}\left(w_{0}, w_{1}\right)=\left\{x \in \Omega: 2^{k} \leq w_{0}(x) / w_{1}(x)<2^{k+1}\right\}, \quad k \in \mathbb{Z},
$$

and the powers and weights are related by $1 / p=(1-\theta) / p_{0}+\theta / p_{1}, 1 / q=$ $(1-\theta) / q_{0}+\theta / q_{1}$ and $w=w_{0}^{(1-\theta) p / p_{0}} w_{1}^{\theta p / p_{1}}$.

Proof. We rewrite the space $L$ in the form

$$
\begin{aligned}
L & =\left(L_{p_{0}, q_{0}}\left(w_{0} d \mu\right), L_{p_{1}, q_{1}}\left(w_{1} d \mu\right)\right)_{\theta, q} \\
& =\left(L_{p_{0}, q_{0}}\left(\frac{w_{0}}{w_{1}} d \nu\right), L_{p_{1}, q_{1}}(d \nu)\right)_{\theta, q}, \quad d \nu=w_{1} d \mu .
\end{aligned}
$$

Now we use Theorem 6.1 with $\alpha_{0}=\alpha_{1}=\delta_{1}=0, \delta_{0}=1, w=w_{0} / w_{1}$ and measure $\nu$ instead of $\mu$, to obtain

$$
\begin{aligned}
\|f\|_{L} & \approx\left(\sum_{k \in \mathbb{Z}}\left(\left\|f \chi_{\Omega_{k}}\right\|_{L_{p, q}\left(\left(w_{0} / w_{1}\right)^{\left.(1-\theta) p / p_{0} d \nu\right)}\right.}\right)^{q}\right)^{1 / q} \\
& =\left(\sum_{k \in \mathbb{Z}}\left(\left\|f \chi_{\Omega_{k}}\right\|_{L_{p, q}(w d \mu)}\right)^{q}\right)^{1 / q},
\end{aligned}
$$

where $\Omega_{k}$ is as in the statement.

Counterexample 6.3. Let us show that in general the formula

$$
\left(L_{p_{0}, q_{0}}\left(w_{0} d \mu\right), L_{p_{1}, q_{1}}\left(w_{1} d \mu\right)\right)_{\theta, q}=L_{p, q}(w d \mu)
$$

is not true. Here $1 / p=(1-\theta) / p_{0}+\theta / p_{1}, 1 / q=(1-\theta) / q_{0}+\theta / q_{1}$ and $w=w_{0}^{(1-\theta) p / p_{0}} w_{1}^{\theta p / p_{1}}$.

For this we consider $\Omega=\mathbb{N}$ with the measure $\mu(\{n\})=1$ and the weights $w_{0}(n)=2^{n \theta p / p_{1}}, w_{1}(n)=2^{-n(1-\theta) p / p_{0}}$. Then $w_{0}(n) / w_{1}(n)=2^{n}$, $w(n)=w_{0}(n)^{(1-\theta) p / p_{0}} w_{1}(n)^{\theta p / p_{1}}=1$ and so $\Omega_{k}=\{k\}$, and $w d \mu=d \mu$. Hence, from Corollary 6.2 it follows that

$$
\begin{aligned}
\|f\|_{\left(L_{p_{0}, q_{0}}\left(w_{0} d \mu\right), L_{p_{1}, q_{1}}\left(w_{1} d \mu\right)\right)_{\theta, q}} & \approx\left(\sum_{k \in \mathbb{Z}}\left(\left\|f \chi_{\Omega_{k}}\right\|_{L_{p, q}(d \mu)}\right)^{q}\right)^{1 / q} \\
& =c\left(\sum_{k \in \mathbb{Z}}|f(k)|^{q}\right)^{1 / q}
\end{aligned}
$$

and since

$$
\|f\|_{L_{p, q}(d \mu)} \approx\|\{f(k)\}\|_{l_{p, q}}
$$

it follows that these norms are not equivalent.

Proof of Theorem 6.1. It is enough to prove the theorem under the additional condition

$$
\alpha_{0}=-\alpha_{1}, \quad \delta_{0}=-\delta_{1}
$$


To see this, we set

$$
\widetilde{f}=f \cdot w^{\left(\alpha_{0}+\alpha_{1}\right) / 2}, \quad d \widetilde{\mu}=w^{\left(\delta_{0}+\delta_{1}\right) / 2} d \mu .
$$

Then for $\tilde{f}$ the condition (6.9) and the conditions of Theorem 6.1 are satisfied. Therefore if Theorem 6.1 is true under the additional condition (6.9), then we can apply it to $\widetilde{f}$ and $\widetilde{\mu}$, and coming back to $f$ and $\mu$ we obtain Theorem 6.1 without the restriction (6.9).

So assume that $\alpha_{0}, \alpha_{1}, \delta_{0}$ and $\delta_{1}$ also satisfy (6.9), so that the assumption (6.5) can be written as

$$
\frac{\alpha_{0}}{\delta_{0}}=\frac{\alpha_{1}}{\delta_{1}} \leq 0
$$

Now, we will use the fact that any weighted Lorentz space is an interpolation space between a couple of weighted $L_{p}$-spaces. More precisely, we will find three spaces (on the initial measure space $(\Omega, \mu)$ )

$$
L_{r_{0}}\left(w^{\sigma_{0}}\right), \quad L_{r_{1}}\left(w^{\sigma_{1}}\right), \quad L_{r_{2}}
$$

such that $r_{0} \neq r_{2}, r_{1} \neq r_{2}$ and

$$
L_{p_{i}, q_{i}}\left(w^{\alpha_{i}}, w^{\delta_{i}} d \mu\right)=\left(L_{r_{i}}\left(w^{\sigma_{i}}\right), L_{r_{2}}\right)_{\lambda, q_{i}}, \quad i=0,1 .
$$

Thus the required space $L$ can be expressed in the form

$$
L=\left(\left(L_{r_{0}}\left(w^{\sigma_{0}}\right), L_{r_{2}}\right)_{\lambda, q_{0}},\left(L_{r_{1}}\left(w^{\sigma_{1}}\right), L_{r_{2}}\right)_{\lambda, q_{1}}\right)_{\theta, q} .
$$

By using Theorem 2.3 we see that

$$
L=\left(L_{r_{0}}\left(w^{\sigma_{0}}\right), L_{r_{1}}\left(w^{\sigma_{1}}\right), L_{r_{2}}\right)_{(\theta(1-\lambda), \lambda), q} .
$$

If we apply the description from Theorem 4.1, then we will obtain the required result. This is the plan of the proof. To fulfil it we need first of all to find a triple (6.11) such that the equalities (6.12) are satisfied.

From the Freitag-Lizorkin formula (see [Fr], Th. 1 and Th. 2; [L1], Th. 2 or [L2], Th. 2) it follows that (6.12) will be satisfied if we can solve the system

$$
\begin{aligned}
1 / p_{i} & =(1-\lambda) / r_{i}+\lambda / r_{2}, & & i=0,1, \\
\alpha_{i} & =-\sigma_{i} \frac{r_{i}}{r_{2}-r_{i}}, & i & =0,1, \\
\delta_{i} & =\sigma_{i} r_{2} \frac{r_{i}}{r_{2}-r_{i}}, & i & =0,1,
\end{aligned}
$$

under the restrictions

$$
\lambda \in(0,1), \quad r_{0} \neq r_{2}, \quad r_{1} \neq r_{2}, \quad r_{0}, r_{1}, r_{2}>0, \quad \sigma_{0}, \sigma_{1} \in \mathbb{R} .
$$

To solve this system we first notice that in view of (6.16) the condition (6.17) is equivalent to

$$
\delta_{i}=-\alpha_{i} r_{2}, \quad i=0,1
$$


According to (6.10) we obtain a solution of (6.19) by putting

(6.20) $\quad r_{2}=-\frac{\delta_{0}}{\alpha_{0}}=-\frac{\delta_{1}}{\alpha_{1}}>0 \quad\left(\right.$ if $\alpha_{0}=\alpha_{1}=0$, then we put $\left.r_{2}=\infty\right)$

and so (6.17) will follow from (6.16). Moreover, by the assumption (6.5) in Theorem 6.1 and $(6.20)$, we have

$$
r_{2} \neq p_{i}, \quad i=0,1 \text {. }
$$

Moreover, if (6.16) holds, then

$$
r_{0} \neq r_{2}, \quad r_{1} \neq r_{2}
$$

automatically. Thus we only need to solve the system (6.15) and (6.16), where the unknowns are $\lambda \in(0,1), r_{0}, r_{1}>0$ and $\sigma_{0}, \sigma_{1} \in \mathbb{R}$. This system can be easily solved.

In fact, fix $\lambda \in(0,1)$. Then, in view of $(6.15)$,

$$
\frac{1}{r_{i}}=\frac{1}{1-\lambda}\left(\frac{1}{p_{i}}-\frac{\lambda}{r_{2}}\right), \quad i=0,1,
$$

and the conditions $r_{i}>0(i=0,1)$ will be satisfied if

$$
\lambda<r_{2} / p_{i}, \quad i=0,1 \text {. }
$$

Since $p_{0}$ and $p_{1}$ are finite numbers it is enough to take any $\lambda>0$ which is less than $\min \left(1, r_{2} / p_{0}, r_{2} / p_{1}\right)$. Then the equality (6.16) follows immediately if we choose

$$
\sigma_{i}=-\alpha_{i} \frac{r_{2}-r_{i}}{r_{i}}, \quad i=0,1 .
$$

According to (6.15) we have

$$
1 / p_{i}-1 / r_{2}=(1-\lambda)\left(1 / r_{i}-1 / r_{2}\right)
$$

and by using (6.23) we obtain better formulas for $\sigma_{i}$ and $r_{i}$ :

$$
\begin{aligned}
\sigma_{i} & =-\alpha_{i} \frac{r_{2}-r_{i}}{r_{i}}=\frac{\delta_{i}}{r_{2}} \cdot \frac{r_{2}-r_{i}}{r_{i}}=\delta_{i}\left(\frac{1}{r_{i}}-\frac{1}{r_{2}}\right) \\
& =\delta_{i} \frac{1}{1-\lambda}\left(\frac{1}{p_{i}}-\frac{1}{r_{2}}\right), \quad i=0,1, \\
\frac{1}{r_{i}} & =\frac{1}{r_{2}}+\frac{1}{1-\lambda}\left(\frac{1}{p_{i}}-\frac{1}{r_{2}}\right), \quad i=0,1 .
\end{aligned}
$$

Thus, we have found the following solution for the system (6.15)-(6.18):

(6.26) $r_{2}=-\frac{\delta_{0}}{\alpha_{0}}=-\frac{\delta_{1}}{\alpha_{1}}, \quad \lambda$ any number from $\left(0, \min \left(1, r_{2} / p_{0}, r_{2} / p_{1}\right)\right)$,

$$
\frac{1}{r_{i}}=\frac{1}{r_{2}}+\frac{1}{1-\lambda}\left(\frac{1}{p_{i}}-\frac{1}{r_{2}}\right), \quad i=0,1,
$$




$$
\sigma_{i}=\delta_{i} \frac{1}{1-\lambda}\left(\frac{1}{p_{i}}-\frac{1}{r_{2}}\right), \quad i=0,1 .
$$

The next step will be to apply to the right hand side of (6.14) the description from Theorem 4.1. Thus we need to prove that the points $\left(\sigma_{0}, 1 / r_{0}\right)$, $\left(\sigma_{1}, 1 / r_{1}\right),\left(0,1 / r_{2}\right)$ are not collinear. These points lie on one straight line only if (see (6.27))

$$
\frac{\sigma_{0}}{1 / p_{0}-1 / r_{2}}=\frac{\sigma_{1}}{1 / p_{1}-1 / r_{2}},
$$

but from (6.28) it follows that (6.29) is equivalent to $\delta_{0}=\delta_{1}$, which contradicts the assumption $\delta_{0} \neq \delta_{1}$ in Theorem 6.1.

Therefore we can apply Theorem 4.1 to the right hand side of (6.14) to obtain

$$
\|f\|_{L} \approx\left(\sum_{k \in \mathbb{Z}}\left(\left\|f w^{\sigma} \chi_{\Omega_{k}}\right\|_{L_{r, q}}\right)^{q}\right)^{1 / q},
$$

where $L_{r, q}$ is the usual Lorentz space on $\Omega$ with initial measure $\mu$,

$$
\begin{aligned}
& \sigma=(1-\theta)(1-\lambda) \sigma_{0}+\theta(1-\lambda) \sigma_{1}+\lambda \cdot 0, \\
& \frac{1}{r}=\frac{(1-\theta)(1-\lambda)}{r_{0}}+\frac{\theta(1-\lambda)}{r_{1}}+\frac{\lambda}{r_{2}} .
\end{aligned}
$$

Furthermore, according to (6.28) and (6.26), it follows that

$$
\begin{aligned}
\sigma & =(1-\theta) \delta_{0}\left(\frac{1}{p_{0}}-\frac{1}{r_{2}}\right)+\theta \delta_{1}\left(\frac{1}{p_{1}}-\frac{1}{r_{2}}\right) \\
& =(1-\theta) \frac{\delta_{0}}{p_{0}}+\theta \frac{\delta_{1}}{p_{1}}-\frac{1}{r_{2}}\left((1-\theta) \delta_{0}+\theta \delta_{1}\right) \\
& =(1-\theta) \frac{\delta_{0}}{p_{0}}+\theta \frac{\delta_{1}}{p_{1}}+(1-\theta) \alpha_{0}+\theta \alpha_{1}
\end{aligned}
$$

and, in view of (6.15),

$$
\frac{1}{r}=(1-\theta)\left(\frac{1-\lambda}{r_{0}}+\frac{\lambda}{r_{2}}\right)+\theta\left(\frac{1-\lambda}{r_{1}}+\frac{\lambda}{r_{2}}\right)=\frac{1-\theta}{p_{0}}+\frac{\theta}{p_{1}} .
$$

Thus we have $r=p$ and $\sigma=\sigma_{\alpha}+\sigma_{\delta}$, where

$$
\sigma_{\alpha}=(1-\theta) \alpha_{0}+\theta \alpha_{1}, \quad \sigma_{\delta}=(1-\theta) \frac{\delta_{0}}{p_{0}}+\theta \frac{\delta_{1}}{p_{1}} .
$$

To finish the proof we also need the following simple lemma:

Lemma 6.4. Let $L_{p, q}(\mu)$ be a Lorentz space on $(\Omega, \mu)$. If $c$ is a constant, then

$$
\|c g\|_{L_{p, q}(\mu)}=\|g\|_{L_{p, q}(\nu)}, \quad \nu=c^{p} \mu .
$$


Proof. Indeed, since $g_{\nu}^{*}(t)=g_{\mu}^{*}\left(t / c^{p}\right)$ it follows that

$\|g\|_{L_{p, q}(\nu)}=\left(\int_{0}^{\infty}\left(t^{1 / p} g_{\nu}^{*}(t)\right)^{q} \frac{d t}{t}\right)^{1 / q}=\left(\int_{0}^{\infty}\left(t^{1 / p} g_{\mu}^{*}\left(t / c^{p}\right)\right)^{q} \frac{d t}{t}\right)^{1 / q}=\|c g\|_{L_{p, q}(\mu)}$ and the proof of the lemma is finished.

We continue the proof of Theorem 6.1. Since $w^{\sigma_{\delta}}$ on $\Omega_{k}$ satisfies the inequalities

$$
2^{k \sigma_{\delta}} \leq w(x)^{\sigma_{\delta}} \leq 2^{(k+1) \sigma_{\delta}}, \quad x \in \Omega_{k},
$$

it follows from Lemma 6.4 that up to the constant $2^{\sigma_{\delta}}$ we have

$$
\left\|f w^{\sigma} \chi_{\Omega_{k}}\right\|_{L_{r, q}}=\left\|f w^{\sigma} \chi_{\Omega_{k}}\right\|_{L_{p, q}} \approx\left\|f \chi_{\Omega_{k}}\right\|_{L_{p, q}\left(w^{\sigma_{\alpha}, w^{\sigma} \delta^{p}} d \mu\right)} .
$$

Now using (6.31) and (6.7) we find that

$$
w^{\sigma_{\alpha}}=\left(w^{\alpha_{0}}\right)^{1-\theta}\left(w^{\alpha_{1}}\right)^{\theta}=w^{\alpha} \quad \text { and } \quad w^{\sigma_{\delta} p}=\left[\left(w^{\delta_{0} / p_{0}}\right)^{1-\theta}\left(w^{\delta_{1} / p_{1}}\right)^{\theta}\right]^{p}=w^{\delta} .
$$

The proof is complete.

The restrictions (6.5) are due to the technique used in the proof based on the Second Reiteration Theorem and Theorem 4.1. However, it is not clear if they are really necessary.

Problem 6.5. Are the assumptions (6.5) in Theorem 6.1 necessary?

REMARK 6.6. Note that in the Stein-Weiss theorem the assumption that $p$ is diagonal is essential: see for example Ferreyra $[\mathrm{F}]$, where it has been shown that there exists a positive weight $w$ such that

$$
\left(L_{p_{0}}\left(w^{\alpha_{0}}\right), L_{p_{1}}\left(w^{\alpha_{1}}\right)\right)_{\theta, q} \neq L_{p, q}\left(w^{\alpha}\right) \quad \text { when } q \neq p \text {. }
$$

7. Appendix: proof of Theorem 2.3. First we prove the following lemma of independent interest:

Lemma 7.1. Let $\bar{X}=\left(X_{0}, X_{1}, X_{2}\right)$ be a triple of quasi-Banach spaces. If $0<\alpha_{0}, \alpha_{1}, \mu<1$, then

$$
\left(X_{0}, X_{1}, X_{2}\right)_{\left(\theta_{1}, \theta_{2}\right), 1 ; K} \supset\left(\left(X_{0}, X_{2}\right)_{\alpha_{0}, 1 ; K},\left(X_{1}, X_{2}\right)_{\alpha_{1}, 1 ; K}\right)_{\mu, 1 ; K}
$$

and

$$
\left(X_{0}, X_{1}, X_{2}\right)_{\left(\theta_{1}, \theta_{2}\right), 1 ; J} \subset\left(\left(X_{0}, X_{2}\right)_{\alpha_{0}, 1 ; J},\left(X_{1}, X_{2}\right)_{\alpha_{1}, 1 ; J}\right)_{\mu, 1 ; J}
$$

where $\theta_{1}=\left(1-\alpha_{1}\right) \mu$ and $\theta_{2}=\alpha_{0}(1-\mu)+\alpha_{1} \mu$.

Proof. (a) Let $Y_{i}=\left(X_{i}, X_{2}\right)_{\alpha_{i}, 1 ; K}, i=1,2$. For any $\varepsilon>0$ we take an "almost optimal decomposition" $f=f_{0}+f_{1}$ with $f_{0} \in Y_{0}, f_{1} \in Y_{1}$ and

$$
\left\|f_{0}\right\|_{Y_{0}}+t\left\|f_{1}\right\|_{Y_{1}} \leq(1+\varepsilon) K\left(t, f ; Y_{0}, Y_{1}\right) .
$$


Note that

$$
\begin{aligned}
K\left(t_{1}, t_{2}, f ; X_{0},\right. & \left.X_{1}, X_{2}\right) \\
& \leq C_{2}\left[K\left(t_{2}, f_{0} ; X_{0}, X_{2}\right)+t_{1} K\left(t_{2} / t_{1}, f_{1} ; X_{1}, X_{2}\right)\right] .
\end{aligned}
$$

Indeed, if we take almost optimal decompositions $f_{0}=f_{0}^{0}+f_{2}^{0}$ and $f_{1}=$ $f_{1}^{1}+f_{2}^{1}$ such that

$$
\left\|f_{0}^{0}\right\|_{X_{0}}+t_{2}\left\|f_{2}^{0}\right\|_{X_{2}} \leq(1+\varepsilon) K\left(t_{2}, f_{0} ; X_{0}, X_{2}\right)
$$

and

$$
\left\|f_{1}^{1}\right\|_{X_{1}}+\frac{t_{2}}{t_{1}}\left\|f_{2}^{1}\right\|_{X_{2}} \leq(1+\varepsilon) K\left(\frac{t_{2}}{t_{1}}, f_{1} ; X_{1}, X_{2}\right),
$$

then $f_{0}^{0}+f_{2}^{0}+f_{1}^{1}+f_{2}^{1}=f_{0}+f_{1}=f$ and so

$$
\begin{aligned}
& K\left(t_{1}, t_{2}, f ; X_{0}, X_{1}, X_{2}\right) \\
& \quad \leq\left\|f_{0}^{0}\right\|_{X_{0}}+t_{1}\left\|f_{1}^{1}\right\|_{X_{1}}+t_{2}\left\|f_{2}^{0}+f_{2}^{1}\right\|_{X_{2}} \\
& \quad \leq\left\|f_{0}^{0}\right\|_{X_{0}}+C_{2} t_{2}\left\|f_{2}^{0}\right\|_{X_{2}}+t_{1}\left\|f_{1}^{1}\right\|_{X_{1}}+C_{2} t_{2}\left\|f_{2}^{1}\right\|_{X_{2}} \\
& \quad \leq C_{2}(1+\varepsilon) K\left(t_{2}, f_{0} ; X_{0}, X_{2}\right)+C_{2}(1+\varepsilon) t_{1} K\left(t_{2} / t_{1}, f_{1} ; X_{1}, X_{2}\right) .
\end{aligned}
$$

Since $\varepsilon>0$ was arbitrary we obtain (7.1).

By using (7.1) we obtain

$$
\begin{aligned}
& \|f\|_{\left(\theta_{1}, \theta_{2}\right), 1 ; K}^{\infty} \\
& =\int_{0}^{\infty} \int_{0}^{\infty} t_{1}^{-\theta_{1}} t_{2}^{-\theta_{2}} K\left(t_{1}, t_{2}, f ; \bar{X}\right) \frac{d t_{1}}{t_{1}} \frac{d t_{2}}{t_{2}} \\
& \leq C_{2} \int_{0}^{\infty} \int_{0}^{\infty} t_{1}^{-\theta_{1}} t_{2}^{-\theta_{2}}\left[K\left(t_{2}, f_{0} ; X_{0}, X_{2}\right)+t_{1} K\left(t_{2} / t_{1}, f_{1} ; X_{1}, X_{2}\right)\right] \frac{d t_{1}}{t_{1}} \frac{d t_{2}}{t_{2}} \\
& =C_{2} \int_{0}^{\infty} \int_{0}^{\infty}\left(t_{1}^{1-\alpha_{1}} t_{2}^{\alpha_{1}-\alpha_{0}}\right)^{-\mu}\left[t_{2}^{-\alpha_{0}} K\left(t_{2}, f_{0} ; X_{0}, X_{2}\right)\right] \frac{d t_{1}}{t_{1}} \frac{d t_{2}}{t_{2}} \\
& \quad+C_{2} \int_{0}^{\infty} \int_{0}^{\infty}\left(t_{1}^{1-\alpha_{1}} t_{2}^{\alpha_{1}-\alpha_{0}}\right)^{1-\mu}\left[\left(t_{2} / t_{1}\right)^{-\alpha_{1}} K\left(t_{2} / t_{1}, f_{1} ; X_{1}, X_{2}\right)\right] \frac{d t_{1}}{t_{1}} \frac{d t_{2}}{t_{2}} \\
& =C_{2}\left(I_{1}+I_{2}\right) .
\end{aligned}
$$

In the first integral we make the change of variables

$$
\tau_{1}=t_{1}^{1-\alpha_{1}} t_{2}^{\alpha_{1}-\alpha_{0}}, \quad \tau_{2}=t_{2}
$$

with Jacobian $J\left(\tau_{1}, \tau_{2}\right)=\left(1-\alpha_{1}\right)^{-1} \tau_{1}^{\alpha_{1} /\left(1-\alpha_{1}\right)} \tau_{2}^{-\left(\alpha_{1}-\alpha_{0}\right) /\left(1-\alpha_{1}\right)}$ and so 


$$
\begin{aligned}
I_{1} & =\left(1-\alpha_{1}\right)^{-1} \int_{0}^{\infty} \int_{0}^{\infty} \tau_{1}^{-\mu} \tau_{2}^{-\alpha_{0}} K\left(\tau_{2}, f_{0} ; X_{0}, X_{2}\right) \frac{d \tau_{1}}{\tau_{1}} \frac{d \tau_{2}}{\tau_{2}} \\
& =\left(1-\alpha_{1}\right)^{-1} \int_{0}^{\infty} \tau_{1}^{-\mu}\left\|f_{0}\right\|_{\left(X_{0}, X_{2}\right)_{\alpha_{0}, 1 ; K}} \frac{d \tau_{1}}{\tau_{1}} .
\end{aligned}
$$

In the second integral the change of variables

$$
\tau_{1}=t_{1}^{1-\alpha_{1}} t_{2}^{\alpha_{1}-\alpha_{0}}, \quad \tau_{2}=t_{2} / t_{1}
$$

with Jacobian $J\left(\tau_{1}, \tau_{2}\right)=\left(1-\alpha_{0}\right)^{-1} \tau_{1}^{\left(1+\alpha_{0}\right) /\left(1-\alpha_{0}\right)} \tau_{2}^{2\left(\alpha_{0}-\alpha_{1}\right) /\left(1-\alpha_{0}\right)}$ gives

$$
\begin{aligned}
I_{2} & =\left(1-\alpha_{0}\right)^{-1} \int_{0}^{\infty} \int_{0}^{\infty} \tau_{1}^{1-\mu} \tau_{2}^{-\alpha_{1}} K\left(\tau_{2}, f_{1} ; X_{1}, X_{2}\right) \frac{d \tau_{1}}{\tau_{1}} \frac{d \tau_{2}}{\tau_{2}} \\
& =\left(1-\alpha_{0}\right)^{-1} \int_{0}^{\infty} \tau_{1}^{1-\mu}\left\|f_{1}\right\|_{\left(X_{1}, X_{2}\right)_{\alpha_{1}, 1 ; K}} \frac{d \tau_{1}}{\tau_{1}} .
\end{aligned}
$$

Thus, setting $\gamma=C_{2} \max \left(\left(1-\alpha_{1}\right)^{-1},\left(1-\alpha_{0}\right)^{-1}\right)$, we have

$$
\begin{aligned}
\|f\|_{\left(\theta_{1}, \theta_{2}\right), 1 ; K} & \leq \gamma \int_{0}^{\infty} \tau_{1}^{-\mu}\left[\left\|f_{0}\right\|_{\left(X_{0}, X_{2}\right)_{\alpha_{0}, 1 ; K}}+\tau_{1}\left\|f_{1}\right\|_{\left(X_{1}, X_{2}\right)_{\alpha_{1}, 1 ; K}}\right] \frac{d \tau_{1}}{\tau_{1}} \\
& \left.=\gamma \int_{0}^{\infty} \tau_{1}^{-\mu}\left[\left\|f_{0}\right\|_{Y_{0}}+\tau_{1}\left\|f_{1}\right\|_{Y_{1}}\right] \frac{d \tau_{1}}{\tau_{1}} \quad \text { [by }(7.1)\right] \\
& \leq \gamma(1+\varepsilon) \int_{0}^{\infty} \tau_{1}^{-\mu} K\left(\tau_{1}, f ; Y_{0}, Y_{1}\right) \frac{d \tau_{1}}{\tau_{1}}
\end{aligned}
$$

which shows the required imbedding.

(b) Let $Z_{i}=\left(X_{i}, X_{2}\right)_{\alpha_{i}, 1 ; J}, i=1,2$. Assume $f \in\left(X_{0}, X_{1}, X_{2}\right)_{\left(\theta_{1}, \theta_{2}\right), 1 ; J}$. Then $f$ can be represented in the form

$$
\left.f=\int_{0}^{\infty} \int_{0}^{\infty} u\left(t_{1}, t_{2}\right) \frac{d t_{1}}{t_{1}} \frac{d t_{2}}{t_{2}} \quad \text { (convergence in } X_{0}+X_{1}+X_{2}\right),
$$

where $u\left(t_{1}, t_{2}\right)$ is a strongly measurable $X_{0} \cap X_{1} \cap X_{2}$-valued function and satisfies

$$
\left(\int_{0}^{\infty} \int_{0}^{\infty}\left(t_{1}^{-\theta_{1}} t_{2}^{-\theta_{2}} J\left(t_{1}, t_{2}, u\left(t_{1}, t_{2}\right) ; \bar{X}\right)\right)^{q} \frac{d t_{1}}{t_{1}} \frac{d t_{2}}{t_{2}}\right)^{1 / q} \leq(1+\varepsilon)\|f\|_{\left(\theta_{1}, \theta_{2}\right), 1 ; J} .
$$

Put

$$
v\left(t_{1}\right)=\frac{1}{1-\alpha_{1}} \int_{0}^{\infty} u\left(t_{1}^{1 /\left(1-\alpha_{1}\right)} t_{2}^{\left(\alpha_{0}-\alpha_{1}\right) /\left(1-\alpha_{1}\right)}, t_{2}\right) \frac{d t_{2}}{t_{2}}
$$


Then

$$
f=\int_{0}^{\infty} v\left(t_{1}\right) \frac{d t_{1}}{t_{1}} .
$$

In fact, the change of variables

$$
\tau_{1}=t_{1}^{1 /\left(1-\alpha_{1}\right)} t_{2}^{\left(\alpha_{0}-\alpha_{1}\right) /\left(1-\alpha_{1}\right)}, \quad \tau_{2}=t_{2}
$$

has Jacobian $J\left(\tau_{1}, \tau_{2}\right)=\left(1-\alpha_{1}\right) \tau_{1}^{-\alpha_{1}} \tau_{2}^{\alpha_{1}-\alpha_{0}}$ and so

$$
\begin{aligned}
\int_{0}^{\infty} v\left(t_{1}\right) \frac{d t_{1}}{t_{1}} & =\frac{1}{1-\alpha_{1}} \int_{0}^{\infty} \int_{0}^{\infty} u\left(t_{1}^{1 /\left(1-\alpha_{1}\right)} t_{2}^{\left(\alpha_{0}-\alpha_{1}\right) /\left(1-\alpha_{1}\right)}, t_{2}\right) \frac{d t_{1}}{t_{1}} \frac{d t_{2}}{t_{2}} \\
& =\int_{0}^{\infty} \int_{0}^{\infty} u\left(\tau_{1}, \tau_{2}\right) \frac{d \tau_{1}}{\tau_{1}} \frac{d \tau_{2}}{\tau_{2}}=f .
\end{aligned}
$$

Hence, by the definition of the space $\left(Z_{0}, Z_{1}\right)_{\mu, 1 ; J}$ and the $J$-functional we obtain

$$
\begin{aligned}
\|f\|_{\left(Z_{0}, Z_{1}\right)_{\mu, 1 ; J}} & \leq \int_{0}^{\infty} t_{1}^{-\mu} J\left(t_{1}, v\left(t_{1}\right) ; Z_{0}, Z_{1}\right) \frac{d t_{1}}{t_{1}} \\
& \leq \int_{0}^{\infty} t_{1}^{-\mu}\left\|v\left(t_{1}\right)\right\|_{Z_{0}} \frac{d t_{1}}{t_{1}}+\int_{0}^{\infty} t_{1}^{1-\mu}\left\|v\left(t_{1}\right)\right\|_{Z_{1}} \frac{d t_{1}}{t_{1}}=J_{1}+J_{2} .
\end{aligned}
$$

To estimate the first integral we use the definition of the norm in $Z_{0}=$ $\left(X_{0}, X_{2}\right)_{\alpha_{0}, 1 ; J}$ and the representation (7.2) to get

$$
\begin{aligned}
J_{1}= & \int_{0}^{\infty} t_{1}^{-\mu}\left\|v\left(t_{1}\right)\right\|_{Z_{0}} \frac{d t_{1}}{t_{1}} \\
\leq & \frac{1}{1-\alpha_{1}} \int_{0}^{\infty} \int_{0}^{\infty} t_{1}^{-\mu} t_{2}^{-\alpha_{0}} \\
& \times J\left(t_{2}, u\left(t_{1}^{1 /\left(1-\alpha_{1}\right)} t_{2}^{\left(\alpha_{0}-\alpha_{1}\right) /\left(1-\alpha_{1}\right)}, t_{2}\right) ; X_{0}, X_{2}\right) \frac{d t_{2}}{t_{2}} \frac{d t_{1}}{t_{1}} \\
\leq & \frac{1}{1-\alpha_{1}} \int_{0}^{\infty} \int_{0}^{\infty} t_{1}^{-\mu} t_{2}^{-\alpha_{0}}\left[\left\|u\left(t_{1}^{1 /\left(1-\alpha_{1}\right)} t_{2}^{\left(\alpha_{0}-\alpha_{1}\right) /\left(1-\alpha_{1}\right)}, t_{2}\right)\right\|_{X_{0}}\right. \\
& \left.+t_{2}\left\|u\left(t_{1}^{1 /\left(1-\alpha_{1}\right)} t_{2}^{\left(\alpha_{0}-\alpha_{1}\right) /\left(1-\alpha_{1}\right)}, t_{2}\right)\right\|_{X_{2}}\right] \frac{d t_{2}}{t_{2}} \frac{d t_{1}}{t_{1}} .
\end{aligned}
$$

Changing variables

$$
\tau_{1}=t_{1}^{1 /\left(1-\alpha_{1}\right)} t_{2}^{\left(\alpha_{0}-\alpha_{1}\right) /\left(1-\alpha_{1}\right)}, \quad \tau_{2}=t_{2},
$$


with Jacobian $J\left(\tau_{1}, \tau_{2}\right)=\left(1-\alpha_{1}\right) \tau_{1}^{-\alpha_{1}} \tau_{2}^{\alpha_{1}-\alpha_{0}}$, we obtain

$$
\begin{aligned}
J_{1} & \leq \int_{0}^{\infty} \int_{0}^{\infty} \tau_{1}^{-\theta_{1}} \tau_{2}^{-\theta_{2}}\left[\left\|u\left(\tau_{1}, \tau_{2}\right)\right\|_{X_{0}}+\tau_{2}\left\|u\left(\tau_{1}, \tau_{2}\right)\right\|_{X_{2}}\right] \frac{d \tau_{2}}{\tau_{2}} \frac{d \tau_{1}}{\tau_{1}} \\
& \leq 2 \int_{0}^{\infty} \int_{0}^{\infty} \tau_{1}^{-\theta_{1}} \tau_{2}^{-\theta_{2}} J\left(\tau_{1}, \tau_{2}, u\left(\tau_{1}, \tau_{2}\right) ; X_{0}, X_{1}, X_{2}\right) \frac{d \tau_{2}}{\tau_{2}} \frac{d \tau_{1}}{\tau_{1}} \\
& \leq 2(1+\varepsilon)\|f\|_{\left(\theta_{1}, \theta_{2}\right), 1 ; J} .
\end{aligned}
$$

The second integral can be estimated similarly. First we rewrite the representation (7.2) in the form

$$
\begin{aligned}
v\left(t_{1}\right) & =\frac{1}{1-\alpha_{1}} \int_{0}^{\infty} u\left(t_{1}^{1 /\left(1-\alpha_{1}\right)} s_{2}^{\left(\alpha_{0}-\alpha_{1}\right) /\left(1-\alpha_{1}\right)}, s_{2}\right) \frac{d s_{2}}{s_{2}} \\
& =\frac{1}{1-\alpha_{0}} \int_{0}^{\infty} u\left(t_{1}^{1 /\left(1-\alpha_{0}\right)} t_{2}^{\left(\alpha_{0}-\alpha_{1}\right) /\left(1-\alpha_{0}\right)}, t_{1}^{1 /\left(1-\alpha_{0}\right)} t_{2}^{\left(1-\alpha_{1}\right) /\left(1-\alpha_{0}\right)}\right) \frac{d t_{2}}{t_{2}}
\end{aligned}
$$

and then

$$
\begin{aligned}
J_{2}= & \int_{0}^{\infty} t_{1}^{1-\mu}\left\|v\left(t_{1}\right)\right\|_{Z_{1}} \frac{d t_{1}}{t_{1}} \\
\leq & \frac{1}{1-\alpha_{0}} \int_{0}^{\infty} \int_{0}^{\infty} t_{1}^{1-\mu} t_{2}^{-\alpha_{1}} \\
& \times J\left(t_{2}, u\left(t_{1}^{1 /\left(1-\alpha_{0}\right)} t_{2}^{\left(\alpha_{0}-\alpha_{1}\right) /\left(1-\alpha_{0}\right)},\right.\right. \\
\leq & \left.\left.\frac{1}{1-\alpha_{0}} \int_{0}^{\infty} \int_{0}^{\infty} t_{1}^{1-\mu} t_{2}^{-\alpha_{1}} t_{2}^{1 /\left(1-\alpha_{0}\right)} t^{\left(1-\alpha_{1}\right) /\left(1-\alpha_{0}\right)}\right) ; X_{1}, X_{2}\right) \frac{d t_{2}}{t_{2}} \frac{d t_{1}}{t_{1}} \\
& \times\left[\left\|u\left(t_{1}^{1 /\left(1-\alpha_{0}\right)} t_{2}^{\left(\alpha_{0}-\alpha_{1}\right) /\left(1-\alpha_{0}\right)}, t_{1}^{1 /\left(1-\alpha_{0}\right)} t_{2}^{\left(1-\alpha_{1}\right) /\left(1-\alpha_{0}\right)}\right)\right\|_{X_{1}}\right. \\
& \left.+t_{2}\left\|u\left(t_{1}^{1 /\left(1-\alpha_{0}\right)} t_{2}^{\left(\alpha_{0}-\alpha_{1}\right) /\left(1-\alpha_{0}\right)}, t_{1}^{1 /\left(1-\alpha_{0}\right)} t_{2}^{\left(1-\alpha_{1}\right) /\left(1-\alpha_{0}\right)}\right)\right\|_{X_{2}}\right] \frac{d t_{2}}{t_{2}} \frac{d t_{1}}{t_{1}} .
\end{aligned}
$$

Changing variables

$$
\tau_{1}=t_{1}^{1 /\left(1-\alpha_{0}\right)} t_{2}^{\left(\alpha_{0}-\alpha_{1}\right) /\left(1-\alpha_{0}\right)}, \quad \tau_{2}=t_{1}^{1 /\left(1-\alpha_{0}\right)} t_{2}^{\left(1-\alpha_{1}\right) /\left(1-\alpha_{0}\right)},
$$

with Jacobian $J\left(\tau_{1}, \tau_{2}\right)=\left(1-\alpha_{0}\right) \tau_{1}^{-\alpha_{1}} \tau_{2}^{\alpha_{1}-\alpha_{0}}$, we obtain

$$
J_{2} \leq \int_{0}^{\infty} \int_{0}^{\infty} \tau_{1}^{-\theta_{1}} \tau_{2}^{-\theta_{2}} \tau_{1}\left[\left\|u\left(\tau_{1}, \tau_{2}\right)\right\|_{X_{1}}+\frac{\tau_{2}}{\tau_{1}}\left\|u\left(\tau_{1}, \tau_{2}\right)\right\|_{X_{2}}\right] \frac{d \tau_{2}}{\tau_{2}} \frac{d \tau_{1}}{\tau_{1}}
$$




$$
\begin{aligned}
& \leq 2 \int_{0}^{\infty} \int_{0}^{\infty} \tau_{1}^{-\theta_{1}} \tau_{2}^{-\theta_{2}} J\left(\tau_{1}, \tau_{2}, u\left(\tau_{1}, \tau_{2}\right) ; X_{0}, X_{1}, X_{2}\right) \frac{d \tau_{2}}{\tau_{2}} \frac{d \tau_{1}}{\tau_{1}} \\
& \leq 2(1+\varepsilon)\|f\|_{\left(\theta_{1}, \theta_{2}\right), 1 ; J .}
\end{aligned}
$$

By putting together all the above estimates we obtain

$$
\|f\|_{\left(Z_{0}, Z_{1}\right)_{\mu, 1 ; J}} \leq 4(1+\varepsilon)\|f\|_{\left(\theta_{1}, \theta_{2}\right), 1 ; J}
$$

and the lemma is proved.

REMARK 7.2. In view of our proof above we see that Lemma 7.1 is true even for triples of quasi-normed Abelian groups.

Proof of Theorem 2.3. By using the power theorem for quasi-Banach couples (see [BL], Th. 3.11.6) we have

$$
\left[\left(\left(X_{0}, X_{2}\right)_{\alpha_{0}, q_{0}},\left(X_{1}, X_{2}\right)_{\alpha_{1}, q_{1}}\right)_{\mu, q}\right]^{q}=\left(\left[\left(X_{0}, X_{2}\right)_{\alpha_{0}, q_{0}}\right]^{q_{0}},\left[\left(X_{1}, X_{2}\right)_{\alpha_{1}, q_{1}}\right]^{q_{0}}\right)_{\eta, 1},
$$

where $\eta=\mu q / q_{1}$ (or equivalently $\left.1-\eta=(1-\mu) q / q_{0}\right)$.

We can find $0<\beta_{0}, \beta_{1}<1$ such that $s_{2}=q_{0} \alpha_{0} / \beta_{0}=q_{1} \alpha_{1} / \beta_{1}$ and we also put $s_{0}=q_{0}\left(1-\alpha_{0}\right) /\left(1-\beta_{0}\right)$ and $s_{1}=q_{1}\left(1-\alpha_{1}\right) /\left(1-\beta_{1}\right)$. We can do this because if, for example, $q_{1} \alpha_{1} \geq q_{0} \alpha_{0}$, then we choose $\beta_{1} \in\left[\beta_{0}, 1\right)$ and take $\beta_{0}=\beta_{1} q_{0} \alpha_{0} /\left(q_{1} \alpha_{1}\right)$. We see that $\beta_{0}<1$. Again by the power theorem for quasi-Banach couples we find that

$$
\left(\left[\left(X_{0}, X_{2}\right)_{\alpha_{0}, q_{0}}\right]^{q_{0}},\left[\left(X_{1}, X_{2}\right)_{\alpha_{1}, q_{1}}\right]^{q_{0}}\right)_{\eta, 1}=\left(\left(X_{0}^{s_{0}}, X_{2}^{s_{2}}\right)_{\beta_{0}, 1},\left(X_{1}^{s_{1}}, X_{2}^{s_{2}}\right)_{\beta_{1}, 1}\right)_{\mu, 1}
$$

By Lemma 7.1 together with the equivalence Theorem 1 of [AK] for quasiBanach function lattices, we find that

$$
\left(\left(X_{0}^{s_{0}}, X_{2}^{s_{2}}\right)_{\beta_{0}, 1},\left(X_{1}^{s_{1}}, X_{2}^{s_{2}}\right)_{\beta_{1}, 1}\right)_{\mu, 1}=\left(X_{0}^{s_{0}}, X_{1}^{s_{1}}, X_{2}^{s_{2}}\right)_{\left(\lambda_{1}, \lambda_{2}\right), 1},
$$

where

$$
\lambda_{1}=\left(1-\beta_{1}\right) \eta \quad \text { and } \quad \lambda_{2}=\beta_{0}(1-\eta)+\beta_{1} \eta .
$$

By using the power theorem of Sparr [S, Th. 7.1] for triples of quasi-normed Abelian groups we obtain

$$
\left(X_{0}^{s_{0}}, X_{1}^{s_{1}}, X_{2}^{s_{2}}\right)_{\left(\lambda_{1}, \lambda_{2}\right), 1}=\left[\left(X_{0}, X_{1}, X_{2}\right)_{\left(\theta_{1}, \theta_{2}\right), q}\right]^{q},
$$

where $\theta_{1}=\lambda_{1} s_{1} / q, \theta_{2}=\lambda_{2} s_{2} / q$ and $1-\theta_{1}-\theta_{2}=\left(1-\lambda_{1}-\lambda_{2}\right) s_{0} / q$. Thus

$$
\left(\left(X_{0}, X_{2}\right)_{\alpha_{0}, q_{0}},\left(X_{1}, X_{2}\right)_{\alpha_{1}, q_{1}}\right)_{\eta, q}=\left(X_{0}, X_{1}, X_{2}\right)_{\left(\theta_{1}, \theta_{2}\right), q}
$$

with equivalent quasi-norms (observe that $\theta_{1}=\lambda_{1} s_{1} / q=\left(1-\alpha_{1}\right) \mu$ and also $\left.\theta_{2}=\lambda_{2} s_{2} / q=\alpha_{0}(1-\mu)+\alpha_{1} \mu\right)$ and Theorem 2.3 is proved.

\section{References}

[A] I. U. Asekritova, A counterexample to K-divisibility for $(n+1)$-tuples of Banach spaces, in: Studies in the Theory of Functions of Several Real Variables, Yaroslavl', 1988, 5-15 (in Russian). 
[AK] I. Asekritova and N. Krugljak, On equivalence of $K$ - and J-methods for $(n+1)$ tuples of Banach spaces, Studia Math. 122 (1997), 99-116.

[BS] A. Baernstein II and E. T. Sawyer, Embedding and multiplier theorems for $H^{p}\left(R^{n}\right)$, Mem. Amer. Math. Soc. 318 (1985).

[BL] J. Bergh and J. Löfström, Interpolation Spaces. An Introduction, Springer, Berlin, 1976.

[BK] Yu. A. Brudnyı̌ and N. Ya. Krugljak, Interpolation Functors and Interpolation Spaces, North-Holland, Amsterdam, 1991.

[CFMR] F. Cobos, P. Fernandez-Martinez, A. Martínez and Y. Raynaud, On duality between $K$ - and J-spaces, Proc. Edinburgh Math. Soc. 42 (1999), 43-63.

[CJ] M. Cwikel and S. Janson, Real and complex interpolation methods for finite and infinite families of Banach spaces, Adv. Math. 66 (1987), 234-290.

[E] S. Ericsson, Descriptions of some $K$ functionals for three spaces and reiteration, Math. Nachr. 202 (1999), 29-41.

[F] E. V. Ferreyra, On a negative result concerning interpolation with change of measures for Lorentz spaces, Proc. Amer. Math. Soc. 125 (1997), 1413-1417.

[Fr] D. Freitag, Real interpolation of weighted $L_{p}$-spaces, Math. Nachr. 86 (1978), $15-18$.

[G] J. E. Gilbert, Interpolation between weighted $L^{p}$-spaces, Ark. Mat. 10 (1972), $235-249$.

[H] C. Herz, Lipschitz spaces and Bernstein's theorem on absolutely convergent Fourier transforms, J. Math. Mech. 18 (1968), 283-324.

[HJLY] C. Hsiao, B. Jawerth, B. J. Lucier and X. M. Yu, Near optimal compression of orthonormal wavelet expansions, in: Wavelets: Mathematics and Applications, J. J. Benedetto et al. (eds.), CRC Press, Boca Raton, FL, 1994, 425-446.

[JM] B. Jawerth and M. Milman, Wavelets and best approximation in Besov spaces, in: Interpolation Spaces and Related Topics (Haifa, 1990), M. Cwikel et al. (eds.), Israel Math. Conf. Proc. 5, Bar-Ilan Univ., 1992, 107-112.

[KPS] S. G. Kreĭn, Yu. I. Petunin and E. M. Semenov, Interpolation of Linear Operators, Amer. Math. Soc., Providence, 1982.

[Kr] V. L. Krepkogorskiǔ, Interpolation in Lizorkin-Triebel and Besov spaces, Mat. Sb. 185 (1994), No. 7, 63-76 (in Russian); English transl.: Russian Acad. Sci. Sb. Math. 82 (1995), 315-326.

[Ky] G. C. Kyriazis, Wavelet coefficients measuring smoothness in $H_{p}\left(R^{d}\right)$, Appl. Comput. Harmon. Anal. 3 (1996), 100-119.

[LM] J. L. Lions et E. Magenes, Problèmes aux Limites Non Homogènes et Applications I, Springer, Berlin, 1972.

[LP] J. L. Lions et J. Peetre, Sur une classe d'espaces d'interpolation, Inst. Hautes Études Sci. Publ. Math. 19 (1964), 5-68.

[L1] P. I. Lizorkin, Interpolation of weighted $L^{p}$-spaces, Dokl. Akad. Nauk SSSR 222 (1975), 32-35 (in Russian); English transl.: Soviet Math. Dokl. 16 (1975), $577-581$.

[L2] - Interpolation of $L_{p}$-spaces with a weight, Trudy Mat. Inst. Steklov. 140 (1976), 201-211 (in Russian); English transl.: Proc. Steklov Inst. Math. 140 (1979), 221-232.

[M] L. Maligranda, On commutativity of interpolation with intersection, Suppl. Rend. Circ. Mat. Palermo 10 (1985), 113-118.

[MP] L. Maligranda and L. E. Persson, Real interpolation between weighted $L^{p}$ and Lorentz spaces, Bull. Polish Acad. Sci. Math. 35 (1987), 765-778.

[Me] Y. Meyer, Wavelets and Operators, Cambridge Univ. Press, 1992. 
[P] J. Peetre, New Thoughts on Besov Spaces, Duke Univ. Math. Ser., Durham, 1976.

[Pe] L. E. Persson, Descriptions of some interpolation spaces in off-diagonal cases, in: Lecture Notes in Math. 1070, Springer, 1984, 213-231.

[S] G. Sparr, Interpolation of several Banach spaces, Ann. Mat. Pura Appl. 99 (1974), 247-316.

[SW] E. M. Stein and G. Weiss, Interpolation of operators with change of measures, Trans. Amer. Math. Soc. 87 (1958), 159-172.

[Y] A. Yoshikawa, Sur la théorie d'espaces d'interpolation-les espaces de moyenne de plusieurs espaces de Banach, J. Fac. Sci. Univ. Tokyo Sect. I 16 (1970), 407-468.

Irina Asekritova

Department of Mathematics

Yaroslavl' State Pedagogical University

Respublikanskaya 108

150000 Yaroslavl', Russia

E-mail: asekr@yspu.yar.ru

Lech Maligranda and Lars-Erik Persson

Department of Mathematics

Luleå University of Technology

S-971 87 Luleå, Sweden

E-mail: lech@sm.luth.se

larserik@sm.luth.se
Natan Krugljak

Department of Mathematics Yaroslavl' State University

Sovetskaya 14

150000 Yaroslavl', Russia

E-mail: natan@univ.uniyar.ac.ru

Lyudmila Nikolova

Department of Mathematics

Sofia University

blv. J. Bouchier 5

1164 Sofia, Bulgaria

E-mail: ludmilan@fmi.uni-sofia.bg

Received March 7, 2000

Revised version August 31, 2000 